Louisiana State University

LSU Digital Commons

$12-6-2007$

\title{
Recoil velocities from equal-mass binary black-hole mergers: A systematic investigation of spin-orbit aligned configurations
}

\author{
Denis Pollney \\ Max Planck Institute for Gravitational Physics (Albert Einstein Institute) \\ Christian Reisswig \\ Max Planck Institute for Gravitational Physics (Albert Einstein Institute) \\ Luciano Rezzolla \\ Max Planck Institute for Gravitational Physics (Albert Einstein Institute) \\ Béla Szilágyi \\ Max Planck Institute for Gravitational Physics (Albert Einstein Institute) \\ Marcus Ansorg \\ Max Planck Institute for Gravitational Physics (Albert Einstein Institute)
}

See next page for additional authors

Follow this and additional works at: https://digitalcommons.Isu.edu/physics_astronomy_pubs

\section{Recommended Citation}

Pollney, D., Reisswig, C., Rezzolla, L., Szilágyi, B., Ansorg, M., Deris, B., Diener, P., Dorband, E., Koppitz, M., Nagar, A., \& Schnetter, E. (2007). Recoil velocities from equal-mass binary black-hole mergers: A systematic investigation of spin-orbit aligned configurations. Physical Review D - Particles, Fields, Gravitation and Cosmology, 76 (12) https://doi.org/10.1103/PhysRevD.76.124002

This Article is brought to you for free and open access by the Department of Physics \& Astronomy at LSU Digital Commons. It has been accepted for inclusion in Faculty Publications by an authorized administrator of LSU Digital Commons. For more information, please contact ir@lsu.edu. 


\section{Authors}

Denis Pollney, Christian Reisswig, Luciano Rezzolla, Béla Szilágyi, Marcus Ansorg, Barrett Deris, Peter Diener, Ernst Nils Dorband, Michael Koppitz, Alessandro Nagar, and Erik Schnetter 


\title{
Recoil velocities from equal-mass binary black-hole mergers: A systematic investigation of spin-orbit aligned configurations
}

\author{
Denis Pollney, ${ }^{1}$ Christian Reisswig, ${ }^{1}$ Luciano Rezzolla, ${ }^{1,2}$ Béla Szilágyi, ${ }^{1}$ Marcus Ansorg, ${ }^{1}$ Barrett Deris, ${ }^{3,4}$ \\ Peter Diener, ${ }^{2,3}$ Ernst Nils Dorband, ${ }^{1}$ Michael Koppitz, ${ }^{1}$ Alessandro Nagar, ${ }^{5}$ and Erik Schnetter ${ }^{2,3}$ \\ ${ }^{1}$ Max-Planck-Institut für Gravitationsphysik, Albert-Einstein-Institut, Potsdam-Golm, Germany \\ ${ }^{2}$ Department of Physics and Astronomy, Louisiana State University, Baton Rouge, Louisiana, USA \\ ${ }^{3}$ Center for Computation \& Technology, Louisiana State University, Baton Rouge, Louisiana, USA \\ ${ }^{4}$ Department of Physics, University of California at San Diego, La Jolla, California, USA \\ ${ }^{5}$ Dipartimento di Fisica, Politecnico di Torino and INFN, sez. di Torino, Italy.
}

(Received 6 September 2007; published 6 December 2007)

\begin{abstract}
Binary black-hole systems with spins aligned with the orbital angular momentum are of special interest, as studies indicate that this configuration is preferred in nature due to non-vacuum environmental interactions, as well as post-Newtonian (PN) spin-orbit couplings. If the spins of the two bodies differ, there can be a prominent beaming of the gravitational radiation during the late plunge, causing a recoil of the final merged black hole. In this paper we perform an accurate and systematic study of recoil velocities from a sequence of equal-mass black holes whose spins are aligned with the orbital angular momentum, and whose individual spins range from $a=+0.584$ to -0.584 . In this way we extend and refine the results of a previous study which concentrated on the antialigned portion of this sequence, to arrive at a consistent maximum recoil of $448 \pm 5 \mathrm{~km} / \mathrm{s}$ for antialigned models as well as to a phenomenological expression for the recoil velocity as a function of spin ratio. Quite surprisingly, this relation highlights a nonlinear behavior, not predicted by the PN estimates, and can be readily employed in astrophysical studies on the evolution of binary black holes in massive galaxies. An essential result of our analysis, without which no systematic behavior can be found, is the identification of different stages in the waveform, including a transient due to lack of an initial linear momentum in the initial data. Furthermore, by decomposing the recoil computation into coupled modes, we are able to identify a pair of terms which are largely responsible for the kick, indicating that an accurate computation can be obtained from modes up to $\ell=3$. Finally, we provide accurate measures of the radiated energy and angular momentum, finding these to increase linearly with the spin ratio, and derive simple expressions for the final spin and the radiated angular momentum which can be easily implemented in $N$-body simulations of compact stellar systems. Our code is calibrated with strict convergence tests and we verify the correctness of our measurements by using multiple independent methods whenever possible.
\end{abstract}

DOI: 10.1103/PhysRevD.76.124002

PACS numbers: 04.25.Dm, 04.30.Db, 95.30.Sf, 97.60.Lf

\section{INTRODUCTION}

Recent developments in numerical relativity have solved the problem of stably evolving black hole initial data for useful timescales, and opened the door to studies of physical phenomena resulting from strong-field gravitational interactions. A result of particular interest to astrophysics is an accurate calculation of the recoil velocity which is generated during an asymmetric collision of a black-hole binary. It is well known that a binary with unequal masses or spins of the individual bodies will radiate gravitational energy asymmetrically. This results in an uneven flux, which gives a net linear momentum to the final black hole, often called a "kick" [1,2]. While estimations of kick velocities have been available for some time [3-5], the largest part of the system's acceleration is generated in the final orbits of the binary system, and as such requires fully relativistic calculations to be determined accurately.

Over the past year, a number of numerical relativity simulations have been carried out to determine recoil velocities in various sections of the parameter space of binary black-hole systems. The first systems to be studied were unequal mass systems with moderate mass ratios, where the first calculations were performed by the Penn State [6] and Goddard [7] groups, with simulations at mass ratios near the estimated peak of the Fitchett formula [4]. A more extensive study, exploring a large number of models between mass ratios 0.25 to 1.0 , was carried out by the Jena group [8], providing for the first time a mapping of the unequal mass parameter space with fully relativistic simulations. The recoils from systems in which the bodies had spin were first considered by a number of studies in the first half of this year. The Penn State group examined a sequence of equal mass binaries with spins equal and antialigned, determining that the largest recoil possible from such an evolution is of the order of $475 \mathrm{~km} / \mathrm{s}$ [9]. At the same time, in Ref. [10] we studied a sequence of models in which the spins are antialigned, but of different magnitude, and arrived at a similar estimate of $450 \mathrm{~km} / \mathrm{s}$. The Jena and Brownsville (now Rochester) groups showed that extremely large kicks are possible from particular configurations of misaligned spins, measuring recoils as high as $2500 \mathrm{~km} / \mathrm{s} \mathrm{[11]}$, and extrapolating to $4000 \mathrm{~km} / \mathrm{s}$ for the maximally spinning case $[12,13]$. Such spin configurations 
have recently been studied in more detail in [14]. Velocities of this magnitude have a number of astrophysical implications for models of galaxy mergers.

In this paper, we expand on the work performed in [10], extending it in a number of different ways. First, we consider a larger sequence of aligned but unequal spins with spin-ratios ranging from -1 to +1 , where the spins are aligned (or antialigned) with the orbital angular momentum. Our interest in this set of models is motivated by the fact that there are strong indications that binary blackhole systems having spins aligned with the orbital angular momentum are preferred in nature. Post-Newtonian (PN) studies in vacuum have in fact shown that in vacuum the gravitational spin-orbit coupling has a tendency to bring about such an alignment from generic initial conditions [15]. Furthermore, in astrophysical situations where there is likely to be at least some component of interstellar matter inducing a dissipative dynamics, there is also a tendency to align [16].

We describe the influence of the initial dynamics on the radiated waveforms and the importance of suitable vector integration constants to remove these effects when determining the final recoil velocity. These vectors, in fact, capture the information about the net linear momentum that the spacetime has built-up during its past evolution and prior to the actual numerical evolution and can result into a significant correction. We discuss how to use the results obtained to derive a phenomenological expression for the recoil velocity as a function of the spin ratio. Finally, we also compute how the angular momentum of the system is redistributed between radiation and the spin of the final black hole, providing useful expressions as functions of the spin ratio.

The paper is organized as follows: Sec. II describes the code, as well as the initial data construction, and calibration tests. In Sec. III we discuss the calculation of the recoil velocity from gravitational-wave data on a large sphere. We introduce and compare two methods, one based on the Newman-Penrose $\Psi_{4}$ scalar which is the usual method that has been adopted in recent numerical studies, and another which is based on perturbations of Schwarzschild black holes modeled by a gauge-invariant formalism. Though the two methods are based on quite different underlying assumptions, they agree very well in their estimation of physical quantities, and, in particular, the recoil velocity. Section IV describes evolutions of the aligned-spin sequence and the dependence of the recoil velocity on the spin-ratio. We find that the data show an almost linear behavior at large negative spin-ratios, as predicted by PN calculations. However taking into account also results from positive spin-ratios, the data suggest a nonlinear (quadratic) dependence and we give a phenomenological expression for the recoil velocity as a function of the spin ratio. Extrapolating our results to the case of maximally rotating black holes, we find that the maximum recoil velocity attainable by spin-orbit aligned configurations is $448 \pm 5 \mathrm{~km} / \mathrm{s}$. Finally, we discuss the radiation of mass and angular momentum for these evolutions, determining the parameters of the isolated final black holes and show the excellent conservation of mass and angular momentum recorded in our simulations. Again we provide phenomenological expressions for the relative amount of radiated mass and spin as functions of the initial spin ratio.

In the following equations we use Greek indices (running from 0 to 3 ) to denote components of fourdimensional objects and Latin indices (running from 1 to 3 ) for three-dimensional ones that are defined on spacelike foliations of the spacetime.

\section{MATHEMATICAL AND NUMERICAL SETUP}

The data presented in this paper were produced using the CCATIE code, a three-dimensional finite differencing code based on the Cactus Computational Toolkit $[17,18]$. The current code is an evolution of previous versions which implemented an excision method and corotating coordinates [19-21]. The main features of the code, in particular, the evolution equations, remain the same. However, some modifications have been introduced in the gauge evolution to accommodate "moving punctures" which has proven to be an effective way to evolve black hole spacetimes $[22,23]$. This method simply removes any restrictions on movement of the punctures from their initial locations, allowing them to be advected on the grid.

\section{A. Evolution system}

We evolve a conformal-traceless " $3+1$ " formulation of the Einstein equations [19,24-26], in which the spacetime is decomposed into three-dimensional spacelike slices, described by a metric $\gamma_{i j}$, its embedding in the full spacetime, specified by the extrinsic curvature $K_{i j}$, and the gauge functions $\alpha$ (lapse) and $\beta^{i}$ (shift) that specify a coordinate frame (see Sec. II B for details on how we treat gauges and [27] for a general description of the $3+1$ split). The particular system which we evolve transforms the standard ADM variables as follows. The 3-metric $\gamma_{i j}$ is conformally transformed via

$$
\phi=\frac{1}{12} \ln \operatorname{det} \gamma_{i j}, \quad \tilde{\gamma}_{i j}=e^{-4 \phi} \gamma_{i j},
$$

and the conformal factor $\phi$ evolved as an independent variable, whereas $\tilde{\gamma}_{i j}$ is subject to the constraint $\operatorname{det} \tilde{\gamma}_{i j}=$ 1 . The extrinsic curvature is subjected to the same conformal transformation, and its trace $\operatorname{tr} K_{i j}$ evolved as an independent variable. That is, in place of $K_{i j}$ we evolve:

$$
K \equiv \operatorname{tr} K_{i j}=g^{i j} K_{i j}, \quad \tilde{A}_{i j}=e^{-4 \phi}\left(K_{i j}-\frac{1}{3} \gamma_{i j} K\right),
$$

with $\operatorname{tr} \tilde{A}_{i j}=0$. Finally, new evolution variables

$$
\tilde{\Gamma}^{i}=\tilde{\gamma}^{j k} \tilde{\Gamma}_{j k}^{i}
$$


are introduced, defined in terms of the Christoffel symbols of the conformal 3-metric.

The Einstein equations specify a well known set of evolution equations for the listed variables and are given by

$$
\begin{gathered}
\left(\partial_{t}-\mathcal{L}_{\beta}\right) \tilde{\gamma}_{i j}=-2 \alpha \tilde{A}_{i j}, \\
\left(\partial_{t}-\mathcal{L}_{\beta}\right) \phi=-\frac{1}{6} \alpha K, \\
\left(\partial_{t}-\mathcal{L}_{\beta}\right) \tilde{A}_{i j}=e^{-4 \phi}\left[-D_{i} D_{j} \alpha+\alpha R_{i j}\right]^{\mathrm{TF}} \\
+\alpha\left(K \tilde{A}_{i j}-2 \tilde{A}_{i k} \tilde{A}^{k}{ }_{j}\right), \\
\left(\partial_{t}-\mathcal{L}_{\beta}\right) K=-D^{i} D_{i} \alpha+\alpha\left(\tilde{A}_{i j} \tilde{A}^{i j}+\frac{1}{3} K^{2}\right), \\
\partial_{t} \tilde{\Gamma}^{i}=\tilde{\gamma}^{j k} \partial_{j} \partial_{k} \beta^{i}+\frac{1}{3} \tilde{\gamma}^{i j} \partial_{j} \partial_{k} \beta^{k}+\beta^{j} \partial_{j} \tilde{\Gamma}^{i} \\
-\Gamma^{j} \partial_{j} \beta^{i}+\frac{2}{3} \tilde{\Gamma}^{i} \partial_{j} \beta^{j}-2 \tilde{A}^{i j} \partial_{j} \alpha \\
+2 \alpha\left(\tilde{\Gamma}_{j k}^{i} \tilde{A}^{j k}+6 \tilde{A}^{i j} \partial_{j} \phi-\frac{2}{3} \tilde{\gamma}^{i j} \partial_{j} K\right),
\end{gathered}
$$

where $R_{i j}$ is the three-dimensional Ricci tensor, $D_{i}$ the covariant derivative associated with the three metric $\gamma_{i j}$ and "TF" indicates the trace-free part of tensor objects. The Einstein equations also lead to a set of physical constraint equations that are satisfied within each spacelike slice,

$$
\begin{gathered}
\mathcal{H} \equiv R^{(3)}+K^{2}-K_{i j} K^{i j}=0, \\
\mathcal{M}^{i} \equiv D_{j}\left(K^{i j}-\gamma^{i j} K\right)=0,
\end{gathered}
$$

which are usually referred to as Hamiltonian and momentum constraints. Here $R^{(3)}=R_{i j} \gamma^{i j}$ is the Ricci scalar on a three-dimensional time slice. Our specific choice of evolution variables introduces five additional constraints,

$$
\begin{gathered}
\operatorname{det} \tilde{\gamma}_{i j}=1, \\
\operatorname{tr} \tilde{A}_{i j}=0, \\
\tilde{\Gamma}^{i}=\tilde{\gamma}^{j k} \tilde{\Gamma}_{j k}^{i} .
\end{gathered}
$$

Our code actively enforces the algebraic constraints (11) and (12). The remaining constraints, $\mathcal{H}, \mathcal{M}^{i}$, and (13), are not actively enforced, and can be used as monitors of the accuracy of our numerical solution. See [20] for a more comprehensive discussion of the these points.

\section{B. Gauges}

We specify the gauge in terms of the standard ADM lapse function, $\alpha$, and shift vector, $\beta^{a}$ [28]. We evolve the lapse according to the " $1+\log$ " slicing condition:

$$
\partial_{t} \alpha-\beta^{i} \partial_{i} \alpha=-2 \alpha\left(K-K_{0}\right),
$$

where $K_{0}$ is the initial value of the trace of the extrinsic curvature, and equals zero for the maximally sliced initial data we consider here. The shift is evolved using the hyperbolic $\tilde{\Gamma}$-driver condition [20],

$$
\begin{gathered}
\partial_{t} \beta^{i}-\beta^{j} \partial_{j} \beta^{i}=\frac{3}{4} \alpha B^{i}, \\
\partial_{t} B^{i}-\beta^{j} \partial_{j} B^{i}=\partial_{t} \tilde{\Gamma}^{i}-\beta^{j} \partial_{j} \tilde{\Gamma}^{i}-\eta B^{i},
\end{gathered}
$$

where $\eta$ is a parameter which acts as a damping coefficient. The advection terms on the right-hand-sides of these equations were not present in the original definitions of [20], where comoving coordinates were used, but have been added following the experience of [29,30], and are required for correct advection of the puncture in "movingpuncture" evolutions.

\section{Numerical methods}

Spatial differentiation of the evolution variables is performed via straightforward finite-differencing using fourth-order accurate centered stencils for all but the advection terms for each variable, which are upwinded in the direction of the shift. Vertex-centered adaptive meshrefinement (AMR) is employed using nested grids [31,32] with a 2:1 refinement for successive grid levels, and the highest resolution concentrated in the neighborhood of the individual horizons. Individual apparent horizons are located every few time steps during the evolution [33,34].

The time steps on each grid are set by the Courant condition and thus the spatial grid resolution for that level, with the time evolution being carried out using fourthorder accurate Runge-Kutta integration steps. Boundary data for finer grids are calculated with spatial prolongation operators employing 5th-order polynomials, and prolongation in time employing 2 nd-order polynomials. The latter allows a significant memory saving, requiring only three time levels to be stored, with little loss of accuracy due to the long dynamical time scale relative to the typical grid time step.

In the results presented below we have used 8 levels of mesh refinement with finest grid resolutions of $h / M=$ $0.030,0.024$, and 0.018; we will refer to these resolutions as "low", "medium" and "high", respectively. We find that the medium (i.e., $h=0.024 M$ ) fine-grid resolution is typically good enough to accurately represent the dynamics which we are studying here and will be used hereafter as our fiducial resolution. In this case, the wave-zone grid has a resolution of $h=1.536 M$. In addition, when measuring the convergence order (see discussion in Sect. IIE), we have also used a "very-high" resolution of $h / M=$ 0.012 which therefore gives a factor of 2 refinement with respect to the medium resolution; this should be contrasted with similar convergence tests recently discussed in the literature and in which the refinement factor is much smaller.

The finest grids are centered on each black hole, with a radius about $50 \%$ larger than the apparent horizon. A single 
grid resolution covers the region between $r=20 M$ and $r=80 M$, in which our wave extraction is carried out. The outer (coarsest) grid extends to a spatial position which is large compared with the evolution time of the system. In particular, it ranges from $256 \mathrm{M}$ in each coordinate direction for the binaries which merge rapidly, up to $768 M$ for the binaries which inspiral more slowly because of the spin-orbit interaction. In all cases, artificial wavelike boundary conditions are used, and although these are not explicitly constraint-preserving, they do not introduce major violations of the constraints as long as they are placed sufficiently far away from the central black holes (i.e., with a light-crossing time which is large as compared to the time for the merger). Furthermore, for the models considered here, in which all spins are directed along the $z$-axis of our Cartesian grids, it is possible to use a reflection symmetry condition across the $z=0$ plane. Tests against the runs on a full grid show that this symmetry is preserved to a high degree in our simulations (i.e., with differences below $10^{-14}$ ) so that this symmetry boundary has no influence on the dynamics.

\section{Initial data}

The initial data are constructed applying the "puncture" method [35], which uses Bowen-York extrinsic curvature and solves the Hamiltonian constraint equation numerically as in [36].

We have considered a sequence of binaries for which the initial spin of one of the black holes is held fixed at $S_{2} / M^{2}=0.146 e_{z}$, and the spin of the other black hole is $\boldsymbol{S}_{1} / M^{2}=\left(a_{1} / a_{2}\right) \boldsymbol{S}_{2} / M^{2}$, where the spin ratio $a_{1} / a_{2}$ takes the values $-1,-3 / 4, \ldots, 3 / 4,1$, and $M$ is the sum of the black hole masses, $M=M_{1}+M_{2}$. Thus the black hole spins are antialigned when $a_{1} / a_{2}$ is negative and aligned when it is positive. In all cases the initial data parameters are chosen such that the black hole masses are

$$
M_{i}=\sqrt{\frac{A_{i}}{16 \pi}+\frac{4 \pi S_{i}^{2}}{A_{i}}}=\frac{1}{2},
$$

[37,38] where $A_{i}$ is the area of the $i$-th apparent horizon.

For the orbital initial data parameters we use the effective potential method introduced in [39] and extended to spinning configurations in [40]. The effective potential method is a way of choosing the initial data parameters such that the required physical parameters (e.g. masses and spins) are obtained to describe a binary black-hole system on a quasicircular orbit.

The free parameters to be chosen for the puncture initial data are: the puncture coordinate locations $\boldsymbol{C}_{i}$, the puncture mass parameters $m_{i}$, the linear momenta $\boldsymbol{p}_{i}$, and the individual spins $S_{i}$. Since we are interested in quasicircular orbits we work in the zero momentum frame and choose $\boldsymbol{p}_{1}=-\boldsymbol{p}_{2}$ to be orthogonal to $\boldsymbol{C}_{2}-\boldsymbol{C}_{1}$. The physical parameters we want to control are: the black hole mass ratio $M_{1} / M_{2}$, the orbital angular momentum $\boldsymbol{L}=\boldsymbol{C}_{1} \times$
$\boldsymbol{p}_{1}+\boldsymbol{C}_{2} \times \boldsymbol{p}_{2}$ (see for example [39-41]) and the dimensionless spin parameters $a_{i}=S_{i} / M_{i}^{2}$. In order to choose the input parameters that correspond to the desired physical parameters we have to use a nonlinear root finding procedure, since the physical parameters depend nonlinearly on the input parameters and it is not possible to invert the problem analytically.

As detailed in [40], when the black-hole spins are taken as parameters, it is possible to reduce the number of independent input variables, so that at a given separation $\overline{\boldsymbol{C}} \equiv\left|\boldsymbol{C}_{2}-\boldsymbol{C}_{1}\right| / m_{1}$, the independent input parameters are: $\bar{q} \equiv m_{1} / m_{2}$ and the dimensionless magnitude of the linear momentum $p / m_{1}$. Using a Newton-Raphson method, we solve for $\bar{q}$ and $p / m_{1}$ so that $M_{1} / M_{2}=1$ and the system has a given dimensionless orbital angular momentum, $L /(\mu M)$ where $\mu=m_{1} m_{2} / M^{2}$ is the reduced mass. For such a configuration the initial data solver [36] returns a very accurate value for $M_{\mathrm{ADM}}$, which together with the accurate irreducible mass calculated by the apparent horizon finder $[33,42]$ makes it possible to calculate an accurate value of the dimensionless binding energy

$$
E_{b} / \mu=\left(M_{\mathrm{ADM}}-M_{1}-M_{2}\right) / \mu .
$$

The quasicircular initial data parameters are then obtained by finding the minimum in $E_{b} / \mu$ for varying values of $\overline{\boldsymbol{C}}$ while keeping the required orbital angular momentum $L /(\mu M)$ constant.

We chose a fixed orbital angular momentum $L /(\mu M)=$ 3.3 for our quasicircular orbit initial data parameters. This value was chosen to ensure that model $r 0$ would have enough evolution time for an accurate kick measurement, while at the same time model $r 8$ would not require too much evolution time. In order to check the influence of the evolution time before plunge on the kick measurements of the $r 0$ model, we also calculated initial data for a $r 0$ configuration at larger initial separation $r 0 l$ and at smaller initial separation $r 0 s$. The parameters for all the initial data sets are shown in Table I.

Note that the physical mass $M_{i}$ of a single puncture black hole increases when the spin parameter is increased if the mass parameter $m_{i}$ is kept constant. For that reason obtaining $M_{1}=M_{2}$ in general requires that $m_{1} \neq m_{2}$. Even in the case where the spins have the same magnitude but different directions, the two black holes will have different spin-orbit interactions leading to slightly different physical masses if $m_{1}=m_{2}$. For this reason, the initial data for $r 0$ in Table I has slightly different puncture mass parameters $m_{1} \neq m_{2}$. In contrast, in model $r 8$ the black holes have identical spin parameters and thus also the same spin-orbit interaction, resulting in identical mass parameters $m_{1}=m_{2}$.

\section{E. Convergence tests}

As described in Sec. IIC, the finite difference error of the derivative stencils used in the numerical algorithm is 
TABLE I. The puncture initial data parameters defining the binaries: location $\pm x / M$, linear momenta $\pm p / M$, mass parameters $m_{i} / M$, spins $S_{i} / M^{2}$, dimensionless spins $a_{i}$, ADM mass $M_{\mathrm{ADM}}$ measured at infinity, and ADM angular momentum $J_{\mathrm{ADM}}$ computed from Eq. (47). Note that we set $M_{1}=M_{2}=1 / 2$ [cf., Eq. (17)].

\begin{tabular}{lcccccccccc}
\hline \hline Model & $\pm x / M$ & $\pm p / M$ & $m_{1} / M$ & $m_{2} / M$ & \multicolumn{1}{c}{$S_{1} / M^{2}$} & $S_{2} / M^{2}$ & $a_{1}$ & $a_{2}$ & $M_{\mathrm{ADM}} / M^{2} J_{\mathrm{ADM}} / M^{2}$ \\
\hline$r 0$ & 3.0205 & 0.1366 & 0.4011 & 0.4009 & -0.1460 & 0.1460 & -0.5840 & 0.5840 & 0.9856 \\
$r 1$ & 3.1264 & 0.1319 & 0.4380 & 0.4016 & -0.1095 & 0.1460 & -0.4380 & 0.5840 & 0.9855 \\
$r 2$ & 3.2198 & 0.1281 & 0.4615 & 0.4022 & -0.0730 & 0.1460 & -0.2920 & 0.5840 & 0.9856 & 0.86252 \\
$r 3$ & 3.3190 & 0.1243 & 0.4749 & 0.4028 & -0.0365 & 0.1460 & -0.1460 & 0.5840 & 0.9857 & 0.9346 \\
$r 4$ & 3.4100 & 0.1210 & 0.4796 & 0.4034 & 0.0000 & 0.1460 & 0.0000 & 0.5840 & 0.9859 & 0.9712 \\
$r 5$ & 3.5063 & 0.1176 & 0.4761 & 0.4040 & 0.0365 & 0.1460 & 0.1460 & 0.5840 & 0.9862 & 1.007 \\
$r 6$ & 3.5988 & 0.1146 & 0.4638 & 0.4044 & 0.0730 & 0.1460 & 0.2920 & 0.5840 & 0.9864 & 1.044 \\
$r 7$ & 3.6841 & 0.1120 & 0.4412 & 0.4048 & 0.1095 & 0.1460 & 0.4380 & 0.5840 & 0.9867 & 1.081 \\
$r 8$ & 3.7705 & 0.1094 & 0.4052 & 0.4052 & 0.1460 & 0.1460 & 0.5840 & 0.5840 & 0.9872 & 1.117 \\
$r 0 l$ & 4.1924 & 0.1073 & 0.4066 & 0.4065 & -0.1460 & 0.1460 & -0.5840 & 0.5840 & 0.9889 & 0.8997 \\
$r 0 s$ & 2.8186 & 0.1441 & 0.3997 & 0.3994 & -0.1460 & 0.1460 & -0.5840 & 0.5840 & 0.9849 & 0.8123 \\
\hline \hline
\end{tabular}

$O\left(h^{4}\right)$, while the error in the time-interpolation stencils used for mesh refinement boundary points is $O\left(\Delta t^{3}\right)$. Thus the expected theoretical convergence rate is three. However, it is only time-related operations which are at third order, and since the time step which we use is smaller than the grid spacing and much smaller than the dynamical timescales, we can expect that the error coefficient of the leading order term is quite small. Third order convergence is expected during time-periods when the system goes through rapid dynamical changes, such as the plunge or merger.

The proper convergence of the code was established using the binary system $r 0$, for which we have carried out evolutions using 8 levels of mesh refinement with fine grid-spacings of $h / M=0.024,0.018$, and 0.012 (i.e., resolutions medium, high, and "very-high," respectively, where low refers to $h=0.030$ which was deemed to be of insufficient accuracy for the results of this paper). Other refinement levels have resolutions that are half of the next finest grid. The refinement levels on the initial slice are set up to be identical for the three resolutions and their locations and sizes evolve according to the same algorithm in each case.

We focus on the convergence of a number of different aspects of the code. The first of these is the degree of satisfaction of the Einstein equations, which can be partially determined by examining the Hamiltonian and momentum constraints (9) and (10). A more stringent requirement is to evaluate how well the Einstein tensor satisfies the vacuum condition, $G_{\alpha \beta}=0$. For this we define the positive definite quantity

$\boldsymbol{G} \equiv \begin{cases}\sqrt{G_{00}^{2}+G_{01}^{2}+\cdots+G_{33}^{2}} & \text { outside appar. horizons } \\ 0 & \text { inside appar. horizons. }\end{cases}$

In computing norms over the entire grid, we find it useful to mask out the interiors of the horizons, where the error at the puncture locations - which is not expected to converge - can dominate over more relevant errors in the physically observable domain. In order to compute $G_{\alpha \beta}$ we compute the 4-derivatives of the ADM metric, lapse and shift, then construct the 4-derivatives of the 4-metric from which we can compute the Riemann tensor and then finally obtain $G_{\alpha \beta}$. Time-derivatives are taken using three time-levels, centered around the past time-level. Spatial derivatives are taken using fourth-order accurate centered stencils. Thus the finite-difference error in computing $G_{\alpha \beta}$ is $\mathcal{O}\left(\Delta t^{2}\right)$ in time and $\mathcal{O}\left(h^{4}\right)$ in the space dimensions. Effectively we see a minimum of third order accuracy for this quantity, indicating that the coefficient of the $\mathcal{O}\left(\Delta t^{2}\right)$ error term is small compared to the higher-order terms.

Since the metric gradients and hence the truncation errors are the largest near the black-holes, through the $L_{\infty}$ norm of (19) we effectively monitor that the Einstein tensor converges near the horizons for the duration of the evolution. We regard this as a rather stringent test in comparison with the common use of the $L_{2}$ norm, as the latter tends to dilute errors in small regions or 2D surfaces such as grid boundaries, as they are normalized over the entire grid volume. By contrast, the $L_{\infty}$ norm measures the worst error on the grid, which by propagation of error will also suffer if there are any nonconvergent regions on the grid.

This convergence of $\boldsymbol{G}$ is summarized in Fig. 1, which reports the time evolution of the $L_{\infty}$ norm of (19) at the medium and very-high resolutions. Also indicated with dashed and dotted lines are the expression for the $L_{\infty}$ norm of (19) at the very-high resolution when rescaled for third (dotted line) and fourth-order convergence (dashed line).

There is a period at the beginning of the evolutions where the initial data construction prevents fourth-order convergence. This is due to the fact that the initial data is computed by an interpolation of the results of a spectral 


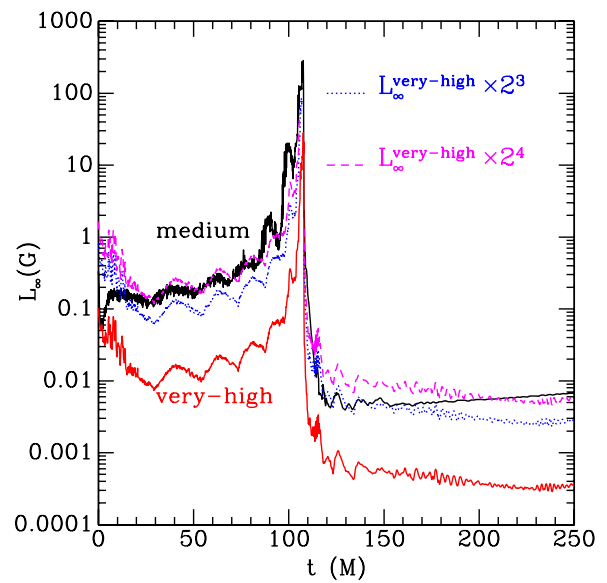

FIG. 1 (color online). The $L_{\infty}$ norm of the Einstein tensor Eq. (19) as a function of time. During the periods of strong dynamics (i.e., when the time derivatives of the evolution variables are large) the convergence order is dominated by the accuracy of the time-interpolation algorithm used at mesh refinement boundaries, thus yielding third-order accuracy. At the times when these time-derivatives are small, the fourth-order finitedifferencing algorithm becomes the dominant source of the error. Note that the very large violations (of $\mathcal{O}(300)$ at the medium resolution) are confined to a single grid point on the trailing edge of the apparent horizon and are produced by the very steep gradients in the shift. As discussed later, this does not affect the fourth-order convergence of the waveforms. At the time of the merger a common apparent horizon forms and its excision from the calculation of the $L_{\infty}$ norm is responsible for the drop in the violation.

solver onto the finite difference grid which is used for evolution. An error is introduced because we keep fixed the number of spectral coefficients and because the Cartesian grid points do not coincide with the spectral collocation points of the Chebyshev polynomials, resulting in a certain amount of high-frequency noise that spoils the convergence for some time at the beginning of the simulation. Numerical dissipation and the constraint damping built into the evolution system implies that the evolution quickly adjusts itself to actually solving the Einstein equations to a good accuracy. The effects of these initial transient modes can last for different amounts of time for the different resolutions, e.g., $\sim 10 M$ for the medium resolution and $\sim 30 M$ for the very-high resolution.

Soon after this transient has disappeared, the code shows the expected fourth-order convergence, with the largest values of the violation found in the vicinity of the apparent horizons, where the gradients in the metric are the steepest. The violations grow rapidly with time as the binary inspirals and the largest values of the violation of the Einstein tensor are seen at the time of the merger, $t \approx 109 M$, with values as large as $\mathcal{O}(300)$. Such violations are essentially confined to a single grid point on the trailing edge of the apparent horizon and are produced by the very steep gradients in the shift. Clearly, violations of this magnitude would not be revealed when looking at the $L_{2}$ norms and are a source of concern. However, as we will show later, such violations do not propagate away from the horizon to affect the fourth-order convergence of the waveforms.

At the time of the merger the excision of a common apparent horizon from the calculation of the $L_{\infty}$ norm is responsible for the decrease by about four orders of the violation. After this, the $L_{\infty}$ do not grow further in time for the very-high resolution simulation, while a modest increase is seen in the simulation run at medium resolution. During this time the code shows a convergence which is between third-order (right after the merger) and fourthorder (during the ringdown).

In addition to convergence in the Einstein tensor, we also validate the correctness of the physically relevant information contained in the waveforms. We do this by computing convergence rate of the waveforms $Q_{22}^{+}, Q_{33}^{+}$, and $Q_{21}^{\times}$using the ratio of the integrated differences between the medium and high resolutions, and the high and very-high resolutions

$$
\rho(Q) \equiv \frac{\sqrt{\int_{u_{1}}^{u_{2}}\left|Q_{0.024}-Q_{0.018}\right|^{2} d u}}{\sqrt{\int_{u_{1}}^{u_{2}}\left|Q_{0.018}-Q_{0.012}\right|^{2} d u}},
$$

where $u \equiv t-r_{\mathrm{E}}$ is the retarded time at a given detector, $Q$ stands for either $Q_{22}^{+}, Q_{33}^{+}$or $Q_{21}^{\times}$and refers to either its amplitude or the phase. As indicated in Eq. (20), the integrals are evaluated over the retarded interval $\left[u_{1}, u_{2}\right]$ which does not include the initial spurious burst of radiation (which we do not expect to converge) but contains otherwise the complete waveform including the ringdown.

Assuming a truncation error $\mathcal{O}\left(h^{p}\right)$ and that the coefficient of this error does not depend on resolution, the function $\rho$ becomes to leading order

$$
\rho=\frac{\left(h_{0.024}\right)^{p}-\left(h_{0.018}\right)^{p}}{\left(h_{0.018}\right)^{p}-\left(h_{0.012}\right)^{p}}
$$

where $h_{0.024}=0.024 M$ and we we underline the importance of having used a full doubling of the resolution between the smallest and largest resolution to improve the accuracy of this estimate over more narrowly spaced resolution steps. In practice, we measure $\rho$ and then solve for the "effective" convergence order $p$ using Eq. (21). A discussion of the details in this procedure are presented in Appendix A alongside with the computed convergence rates for the amplitudes and phases of $Q$ which are found to between two $(\ell=3)$ and four $(\ell=2)$ (cf., Table V).

It should be noted that the above definition of convergence rate naturally results in noninteger values for the exponent $\rho$, even though our methods are explicitly polynomial. This is because the derivation of (21) assumes a coefficient of one in the leading order error term that extrapolates between the resolutions. If the coefficient is in practice different for a given set of resolutions, then a noninteger value results which is larger if the coefficient is 
smaller. As such, values obtained in this way should not be considered literal polynomial extrapolation orders. By "convergence order 3.8" we rather mean that our results are consistent with third-order finite differencing where the leading third-order error coefficient is quite small so that at the given resolutions the convergence appears to be closer to a fourth-order approximation. Very high convergence exponents are a likely indication that the lowest resolution is not in the convergent regime for the measured quantity. Noninteger convergence orders obtained in this way are resolution dependent, and should themselves converge to the lowest order finite difference approximation used in the code in the limit of infinite resolution.

An important property of the waveforms which has emerged when performing these convergence tests is that the dominant source of error is a dephasing which causes the lower resolution evolutions to "lag" behind the higher resolution. This delay is usually rather small and between $0.1 M$ and $0.5 M$, but it is clearly visible when comparing the total amplitude of $Q$ as a function of time. The most important consequence of this error is that it can spoil the convergence tests if not properly taken into account: the residuals errors seem, in fact, to indicate over-convergence. This is shown in the upper panel of Fig. 2, which reports the differences between $Q_{22}^{+}$when computed at different resolutions scaled for fourth-order convergence. Clearly the overlap is rather poor and even indicating that the truncation error is smaller than expected. This is obviously an artifact of the near cancellation of the lowest-order

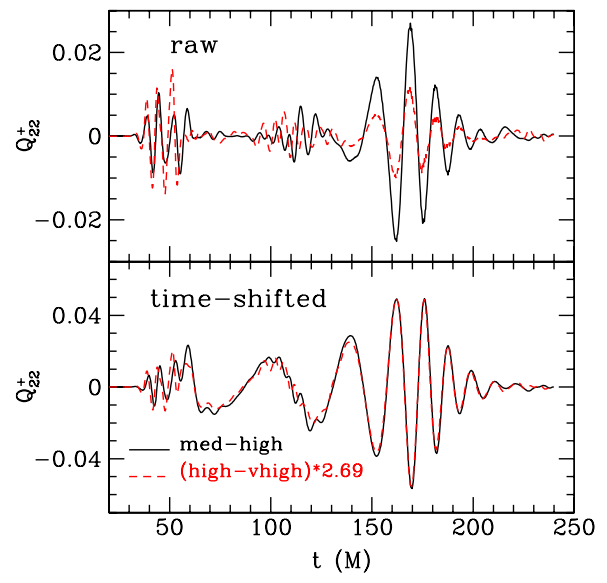

FIG. 2 (color online). Convergence of the fiducial waveform $Q_{22}^{+}$for the binary system $r 0$ before and after the time-shift defined in Eqs. (A1)-(A3). In the upper graph we show the difference between $Q_{22}^{+}$when computed at different resolutions, scaled for fourth-order convergence and using raw data (i.e., without time-shifting). The overlap between the curves is rather poor indicating an over-convergence (i.e., the truncation error appears to be smaller than expected). In the lower panel we show the same data but after time-shifting. The very good overlap of the scaled curves on the indicates that the time-shifting is essential for obtaining properly scaling differences between runs of various resolutions. terms in the truncation error and induced by the small time-differences at different resolutions.

We remove this effect by shifting the time coordinate of the medium and high resolution runs by the time interval needed to produce an alignment of the maxima of the emitted radiation. Details on how to do this are discussed in Appendix A, and we report in the lower panel of Fig. 2 the same data shown in the upper panel, but after the timeshifting. Clearly, the overlap is now extremely good suggesting that the time-shifting is essential for obtaining the expected fourth-order convergence in the waveforms. In accord with the convergence in the waveforms we also see fourth-order convergence in the final kick value.

As a final note we remark that besides validating a proper convergence of the code, it is also important to assess the accuracy of any measurable quantity at the relevant resolutions considered here. As a representative and physically meaningful quantity we have considered the accuracy of the fiducial waveform $Q_{22}^{+}$for the binary system $r 0$. This is shown in Fig. 3, where in the upper graph we report the waveforms at the three different resolutions: very-high (continuous line), high (dashed line) and medium (dotted line). Already with the lowest of these resolutions the accuracy is sufficiently high so that the curves are essentially indistinguishable from each other by eye. The lower panels show magnifications of the relevant portions of the waveform, with the lower-left panel concentrating on the initial transient radiation produced by the truncation error. The latter clearly is rather

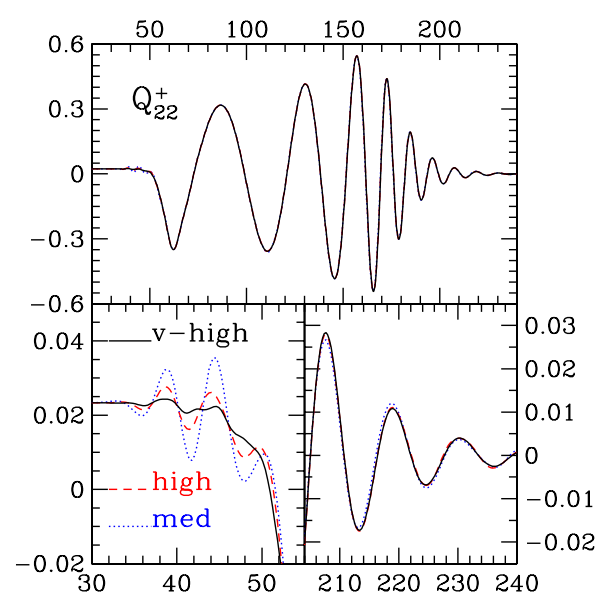

FIG. 3 (color online). Accuracy of the fiducial waveform $Q_{22}^{+}$ for the binary system $r 0$. In the upper graph we show the waveforms at the three different resolutions: very-high (continuous line), high (dashed line), medium (dotted line). The accuracy is very good already with the lowest resolution and the curves cannot be distinguished. The lower panels show magnifications of some relevant portions of the waveform, with the lower-left panel concentrating on the initial transient radiation produced by the truncation error. The lower-right panel, on the other hand, refers to the quasinormal ringing and shows that it is wellcaptured at all resolutions. 
large at the medium resolution, but it nicely converges away when the grid spacing is decreased. The lower-right panel, on the other hand, refers to the quasinormal ringing and shows that it is well-captured at all resolutions.

\section{LINEAR MOMENTUM OF BLACK HOLE SPACETIMES}

In radiating spacetimes where the radiation is emitted asymmetrically, there will be a net linear momentum imparted to the system. In particular, in the case of a binary black hole merger, the final black hole receives a kick which causes it to move off at a given velocity. This velocity can be determined by an analysis of the emitted radiation. In ADM-type numerical simulations, this is typically done by evaluating some scalar quantity which can be associated with the wave energy at some large radius within the computational domain. The chosen radius needs to be large enough that it is in the "wave zone," where nonlinear self-interaction of the gravitational field is negligible and the waves can be picked out as perturbations of a background.

Two methods have become commonplace to determine the emitted wave energy. The first uses the NewmanPenrose curvature scalar $\Psi_{4}$, which can be identified with the gravitational radiation if a suitable frame is chosen at the extraction radius. An alternative method measures the metric of the numerically generated spacetime against a fixed background at the extraction radius, and determines the Zerilli-Moncrief perturbation modes. Both methods yield data for the gravitational wave energy which can be integrated to determine a net linear momentum, as described in more detail in the following sections.

\section{A. Kick measurements via $\boldsymbol{\Psi}_{\mathbf{4}}$}

The Newman-Penrose formalism provides a convenient representation for a number of radiation related quantities as spin-weighted scalars. In particular, the curvature component

$$
\Psi_{4} \equiv-C_{\alpha \beta \gamma \delta} n^{\alpha} \bar{m}^{\beta} n^{\gamma} \bar{m}^{\delta},
$$

is defined as a particular component of the Weyl curvature, $C_{\alpha \beta \gamma \delta}$, projected onto a given null frame, $\{\boldsymbol{l}, \boldsymbol{n}, \boldsymbol{m}, \overline{\boldsymbol{m}}\}$. In practice, we define an orthonormal basis in the three space $(\hat{\boldsymbol{r}}, \hat{\boldsymbol{\theta}}, \hat{\boldsymbol{\phi}})$, centered on the Cartesian grid center and oriented with poles along $\hat{z}$. The normal to the slice defines a timelike vector $\hat{\boldsymbol{t}}$, from which we construct the null frame

$$
\begin{gathered}
\boldsymbol{l}=\frac{1}{\sqrt{2}}(\hat{\boldsymbol{t}}-\hat{\boldsymbol{r}}), \quad \boldsymbol{n}=\frac{1}{\sqrt{2}}(\hat{\boldsymbol{t}}+\hat{\boldsymbol{r}}), \\
\boldsymbol{m}=\frac{1}{\sqrt{2}}(\hat{\boldsymbol{\theta}}-\mathrm{i} \hat{\phi}) .
\end{gathered}
$$

We then calculate $\Psi_{4}$ via a reformulation of (22) in terms of ADM variables on the slice [43],

$$
\Psi_{4}=C_{i j} \bar{m}^{i} \bar{m}^{j},
$$

where

$$
C_{i j} \equiv R_{i j}-K K_{i j}+K_{i}{ }^{k} K_{k j}-\mathrm{i} \epsilon_{i}{ }^{k l} \nabla_{l} K_{j k} .
$$

The identification of the Newman-Penrose $\Psi_{4}$ with the gravitational radiation content of the spacetime is a result of the peeling theorem, which states that in an appropriate frame the $\Psi_{4}$ component of the curvature has the slowest falloff with radius, $\mathcal{O}(1 / r)$. The conditions of this theorem are not satisfied exactly at a small radius and in the chosen frame. While there are proposals for how this situation can be improved [44], we find that beyond $r_{\mathrm{E}} \geq 30 \mathrm{M}$ in fact our measure of $\Psi_{4}$ scales extremely well with the different extraction radii $r_{\mathrm{E}}$, suggesting that the peeling property is satisfied to a reasonable approximation (see Fig. 4).

The gravitational wave polarization amplitudes $h_{+}$and $h_{\times}$are related to $\Psi_{4}$ by [45]

$$
\ddot{h}_{+}-\mathrm{i} \ddot{h}_{\times}=\Psi_{4},
$$

where the double over-dot stands for second-order time derivative. The flux of linear momentum emitted in gravitational waves in the $i$-direction can be computed from the Isaacson's energy-momentum tensor and can be written in terms of the two polarization amplitudes as [5]

$$
\mathcal{F}_{i} \equiv \dot{P}_{i}=\frac{r^{2}}{16 \pi} \int d \Omega n_{i}\left(\dot{h}_{+}^{2}+\dot{h}_{\times}^{2}\right),
$$

where $n_{i}=x_{i} / r$ is the unit radial vector that points from the source to the observer and $d \Omega=\sin \theta d \phi d \theta$ is the line element of our extraction 2-sphere $S^{2}$. Using Eq. (26), this leads to an expression for the momentum flux in terms of $\Psi_{4}$ as it is commonly used in recent numerical relativity

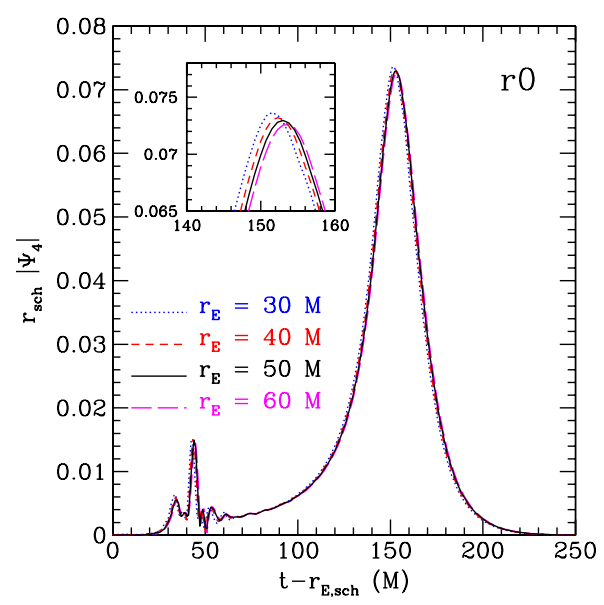

FIG. 4 (color online). Amplitude of $r_{\mathrm{E}, \mathrm{sch}}\left|\Psi_{4}\right|$ for extraction spheres at $r_{\mathrm{E}}=30 M, 40 M, 50 M$, and $60 M$, demonstrating that $\Psi_{4}$ does indeed fall off as required by the peeling property. There is a slight decrease in amplitude with larger radius, suggesting that dissipative effects may become important at larger radii. Results in this paper use waveforms from the $r_{\mathrm{E}}=$ $50 M$ extraction sphere, unless indicated otherwise. 
calculations $[9,11,13,46-49]$ :

$$
\mathcal{F}_{i}=\lim _{r_{\mathrm{sch}} \rightarrow \infty}\left\{\frac{r_{\mathrm{sch}}^{2}}{16 \pi} \int d \Omega n_{i}\left|\int_{-\infty}^{t} d t \Psi_{4}\right|^{2}\right\} .
$$

The Schwarzschild radius, $r_{\mathrm{sch}}$, is derived from the coordinate (isotropic) radius via the standard formula

$$
r_{\text {sch }}=r_{\text {iso }}\left(1-\frac{M}{2 r_{\text {iso }}}\right)^{2} \text {. }
$$

assuming a constant ADM mass $M=M_{\mathrm{ADM}}$ throughout the simulation. With this choice of radial coordinate, expression (28) has been shown to provide recoil velocities which are in better agreement with those obtained through gauge-invariant perturbations than with the alternative coordinate radius, (cf. Sec. III B) and reported in the literature (Additional details on the numerical measurement of $\Psi_{4}$ are presented in Appendix B.)

\section{B. Kick measurements via gauge-invariant perturbations}

An independent method to compute the linear momentum carried away by gravitational radiation is based on the measurements of the nonspherical gauge-invariant perturbations of a Schwarzschild black hole (see Refs. [50-52] for applications to Cartesian coordinates grids). In practice, a set of "observers" is placed on 2-spheres of fixed coordinate radius $r_{\mathrm{E}}$, where they extract the gauge-invariant, odd-parity (or axial) current multipoles $Q_{\ell m}^{\times}$and evenparity (or polar) mass multipoles $Q_{\ell m}^{+}$of the metric perturbation [53]. The numerical implementations of the gauge-invariant variables is done by following the multipolar analysis outlined by Abrahams and Price [54]. The $Q_{\ell m}^{+}$and $Q_{\ell m}^{\times}$variables are related to $h_{+}$and $h_{\times}$as [55]

$$
\begin{aligned}
h_{+}-\mathrm{i} h_{\times}= & \frac{1}{\sqrt{2} r} \sum_{\ell=2}^{\infty} \sum_{m=-\ell}^{\ell}\left(Q_{\ell m}^{+}\right. \\
& \left.-\mathrm{i} \int_{-\infty}^{t} Q_{\ell m}^{\times}\left(t^{\prime}\right) d t^{\prime}\right)_{-2} Y^{\ell m} .
\end{aligned}
$$

Here $-2 Y^{\ell m}$ are the $s=-2$ spin-weighted spherical harmonics and $(\ell, m)$ are the indices of the angular decomposition. Validations of this approach in 3D vacuum spacetimes can be found in Refs. [52,56,57], while its use with matter sources has first been reported in [58].

We note that the notation introduced in Eq. (30) could be misleading as it seems to suggest that $h_{\times}$is always of oddparity and $h_{+}$is always of even-parity. Indeed this is not true in general and in the absence of axisymmetry, i.e., when $m \neq 0$, both $h_{\times}$and $h_{+}$are a superposition of odd and even parity modes. It is only for axisymmetric systems, for which only $m=0$ modes are present, that $Q_{\ell m}^{\times}$and $Q_{\ell m}^{+}$ are real numbers, that $h_{+}$is only even-parity and $h_{\times}$is only odd-parity. Despite this possible confusion, we here prefer to maintain the notation of Eq. (30) which is the most common in the literature [55].
The flux of linear momentum emitted in gravitational waves in terms of $Q_{\ell m}^{+}$and $Q_{\ell m}^{\times}$can be computed by inserting Eq. (30) in Eq. (27), then decomposing $n_{i}$ in spherical harmonics and performing the angular integral. This procedure goes along the lines discussed by Thorne in Ref. [59], where all the relevant formulas are essentially available (cf. Eq. (4.20) there. See also Ref. [60]), so that we only need to adapt them to our notation. In Ref. [59] the even-parity (or electric) multipoles are indicated with $I_{\ell m}$ and the odd-parity (or magnetic) ones with $S_{\ell m}$. They are related to our notation by

$$
\begin{gathered}
{ }^{(\ell)} I_{\ell m}=Q_{\ell m}^{+}, \\
{ }^{(\ell+1)} S_{\ell m}=Q_{\ell m}^{\times},
\end{gathered}
$$

where ${ }^{(\ell)} f_{\ell m} \equiv d^{\ell} f_{\ell m} / d t^{\ell}$. From the well-known property $\left(Q_{\ell m}^{+, \times}\right)^{*}=(-1)^{m} Q_{\ell-m}^{+, \times}$, where the asterisk indicates complex conjugation, one can rewrite Eq. (4.20) of Ref. [59] in a more compact form. Following Ref. [61] where the lowest multipolar contribution was explicitly computed in this way, it is convenient to combine the components of the linear momentum flux in the equatorial plane in a complex number as $\mathcal{F}_{x}+\mathrm{i} \mathcal{F}_{y}$. The multipolar expansion of the flux vector can be written as

$$
\begin{aligned}
\mathcal{F}_{x}+\mathrm{i} \mathcal{F}_{y} & =\sum_{\ell=2}^{\infty} \sum_{m=0}^{\ell} \delta_{m}\left(\mathcal{F}_{x}^{\ell m}+\mathrm{i} \mathcal{F}_{y}^{\ell m}\right), \\
\mathcal{F}_{z} & =\sum_{\ell=2}^{\infty} \sum_{m=0}^{\ell} \delta_{m} \mathcal{F}_{z}^{\ell m},
\end{aligned}
$$

where $\delta_{m}=1$ if $m \neq 0$ and $\delta_{m}=1 / 2$ if $m=0$. Each multipole reads

$$
\begin{aligned}
\mathcal{F}_{x}^{\ell m}+\mathrm{i} \mathcal{F}_{y}^{\ell m} \equiv & \frac{(-1)^{m}}{16 \pi \ell(\ell+1)}\left\{-2 \mathrm{i}\left[a_{\ell m}^{+} \dot{Q}_{\ell-m}^{+} Q_{\ell m-1}^{\times}\right.\right. \\
& \left.+a_{\ell m}^{-} \dot{Q}_{\ell m}^{+} Q_{\ell-(m+1)}^{\times}\right]+\sqrt{\frac{\ell^{2}(\ell-1)(\ell+3)}{(2 \ell+1)(2 \ell+3)}} \\
& \times\left[b_{\ell m}^{-}\left(\dot{Q}_{\ell-m}^{+} \dot{Q}_{\ell+1 m-1}^{+}+Q_{\ell-m}^{\times} \dot{Q}_{\ell+1 m-1}^{\times}\right)\right. \\
& +b_{\ell m}^{+}\left(\dot{Q}_{\ell m}^{+} \dot{Q}_{\ell+1-(m+1)}^{+}\right. \\
& \left.\left.\left.+Q_{\ell m}^{\times} \dot{Q}_{\ell+1-(m+1)}^{\times}\right)\right]\right\} \\
\mathcal{F}_{z}^{\ell m} \equiv & \frac{(-1)^{m}}{8 \pi \ell(\ell+1)}\left\{2 m \operatorname{Im}\left[\dot{Q}_{\ell-m}^{+} Q_{\ell m}^{\times}\right]\right. \\
& +c_{\ell m} \sqrt{\frac{\ell^{2}(\ell-1)(\ell+3)}{(2 \ell+1)(2 \ell+3)}} \operatorname{Re}\left[\dot{Q}_{\ell-m}^{+} Q_{\ell+1 m}^{+}\right. \\
& \left.\left.+Q_{\ell-m}^{\times} \dot{Q}_{\ell+1 m}^{\times}\right]\right\}
\end{aligned}
$$

and 


$$
\begin{gathered}
a_{\ell m}^{ \pm} \equiv \sqrt{(\ell \pm m)(\ell \mp m+1)}, \\
b_{\ell m}^{ \pm} \equiv \sqrt{(\ell \pm m+1)(\ell \pm m+2)}, \\
c_{\ell m} \equiv \sqrt{(\ell-m+1)(\ell-m+1)} .
\end{gathered}
$$

Note that here both $\mathcal{F}_{x}^{\ell m}$ and $\mathcal{F}_{y}^{\ell m}$ are real numbers and are obtained as the real and imaginary part of the right-handside of Eq. (35). For a general system without symmetries one is expecting $\mathcal{F}_{z}^{\ell m}$ to be nonzero. However, our initial data setup, an inspiraling binary with spins antialigned and parallel to the orbital angular momentum, implies that the linear momentum flux vector is completely contained in the equatorial plane of the system and so that $\mathcal{F}_{z}^{\ell m}=0$ by construction. Since we are imposing equatorial symmetry (i.e., invariance for $\theta \rightarrow \pi-\theta$ ) we have that multipoles with $\ell+m=$ even are purely even-parity (i.e., $Q_{\ell m}^{+} \neq 0$ and $\left.Q_{\ell m}^{\times}=0\right)$ and those with $\ell+m=$ odd are purely oddparity (i.e., $Q_{\ell m}^{+}=0$ and $Q_{\ell m}^{\times} \neq 0$ ). As a final remark, we note that for $\ell=m=2$, our Eq. (35) reduces to Eq. (9) of Ref. [61].

\section{RESULTS}

This section collects the results of our analysis of the recoil velocity of spin-aligned binaries and discusses the different aspects of the study which combined provide a consistent and accurate picture of this process. We will first concentrate on the systematic error introduced by the use of initial data with zero linear momentum and on the techniques we have developed to remove it. We will then discuss the actual computation of the recoil velocities and their dependence on the spin ratio, highlighting the modes of the radiation which are largely responsible for the asymmetric emission. Finally, we will discuss the accuracy of our measurements and our ability to preserve mass and angular momentum to below $1 \%$.

\section{A. Initial transients in the waveforms}

Both Eqs. (28) and (35) provide an expression of the recoil velocity in terms of the radiated (linear) momentum per (infinitesimal) time interval. A time-integration of those equations is needed in order to compute the recoil and this obviously opens the question of determining an integration constant which is in practice a vector. Fortunately, this integration constant has here a clear physical meaning and it is therefore easy to compute. In essence it reflects the fact that at the time the simulation is started, the binary system has already accumulated a nonvanishing net momentum as a result of the slow inspiral from an infinite separation.

Since the initial data is constructed so as to have a vanishing linear momentum, there will be a inconsistency between this assumption and the actual evolution of the initial data. Stated differently, the numerical evolution of the Einstein equations will soon tend to a spacetime which is different from the initial one and indeed corresponding to one with a net linear momentum. This momentum is the one that the binary has gained when inspiralling from $t=$ $-\infty$ till $t=0$. Calculating the integration constant amounts therefore to computing the vector accounting for this mismatch and is essential for a correct measurement of the recoil velocity. The error made when neglecting this constant, as routinely done in numerical-relativity calculations, inevitably produces a systematic deviation from the correct answer and, as we will show in the next section, it can altogether prevent from having even the qualitative behavior right.

The relevance of this integration constant depends on the initial separation and it is more important for binaries that start their evolution already quite close. This is rather obvious: the tighter the binary is, the larger the emitted momentum per unit time and the more important is to evaluate the initial mismatch. Figure 5 helps to illustrate this point and can be discussed before entering into the details of how we actually compute the integration constant. The figure shows the time evolution of the recoil velocity $|v|_{\text {kick }} \equiv \sqrt{v_{x}^{2}+v_{y}^{2}}$ for the same binary system having spin ratio $a_{1} / a_{2}=-1$ but with increasing initial separation. More precisely, we consider systems $r 0 l, r 0$ and $r 0 s$ which differ only in the initial separation, which is about 8.4, 6.0, and 5.6M, respectively. The data Fig. 5 is properly shifted in time so as to have the curves overlap and shows that only when the integration constant is properly taken into account, do the three simulations yield the same recoil velocity (cf., solid, dashed, and dotted lines). On the other hand, when the integration constant is not included in the calculation, different evolutions will yield different estimates, with a systematic error that can be as

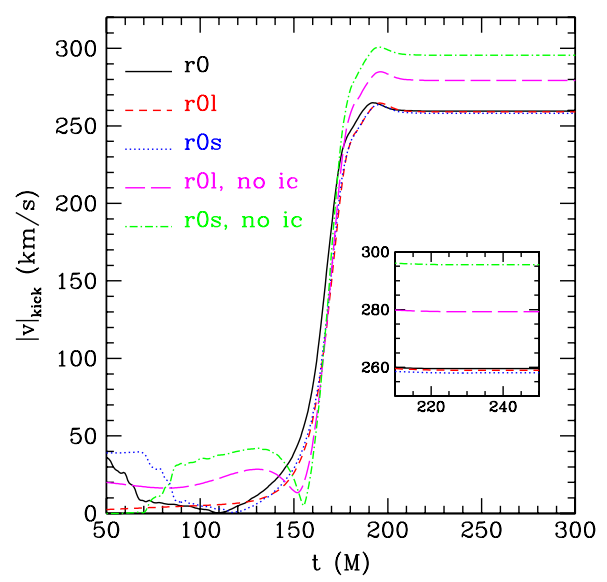

FIG. 5 (color online). The recoil velocity of the binary $r 0$ is compared to those of the same system but with either a larger or a smaller initial separation (i.e., $r 0 l$ and $r 0 s$, respectively). Note the same recoil velocity is obtained when the integration constant is properly taken into account, while an error as large as $\sim 13 \%$ is made otherwise. 
large as $13 \%$ (cf., long-dashed and dot-dashed lines) and is clearly unacceptable given that the overall precision of the simulations is below 1\% (cf., Figs. 11 and 12 and the discussion in Sec. IV D).

Besides providing the right answer, the calculation of the integration constant also results in a considerable saving in computational costs. The complete dynamics of the binary $r 0 l$ including the merger and ringdown, in fact, requires simulations for about $600 \mathrm{M}$; the same answer in terms of recoil velocity can be obtained with the system $r 0 s$, whose dynamics is fully accounted for with a simulation lasting only for $340 M$.

Having stressed the importance of including the integration constant in the measurement of the recoil velocity, we next illustrate how to actually compute it. In essence, it is sufficient to look carefully at the evolution in the velocityspace of the two components $v_{x}$ and $v_{y}$ of the recoil velocity (because of the symmetry the $z$-component is zero but the method described here can be easily extended to the case in which $v^{z} \neq 0$ ). This is shown in the left panel of Fig. 6, which reports the track of the "center of mass" for system $r 0$ in such a space. Different types of line refer to different intervals in time during the evolution and, for an observer at $r_{\mathrm{E}}=50 \mathrm{M}$, the dotted one refers to $t \lesssim 50 \mathrm{M}$, the dashed one to $50 M \lessgtr t \lesssim 75 M$, the continuous one to $75 M \lessgtr t \lesssim 183 M$, and finally the long-dashed one to $t \gtrsim$ $183 M$.

Clearly, for $t \lesssim 50 M$ the system undergoes very little evolution in velocity-space (cf., dotted line in the inset within the inset of the left panel) but a rapid change, lasting for about $25 M$, takes place as the radiation reaches the

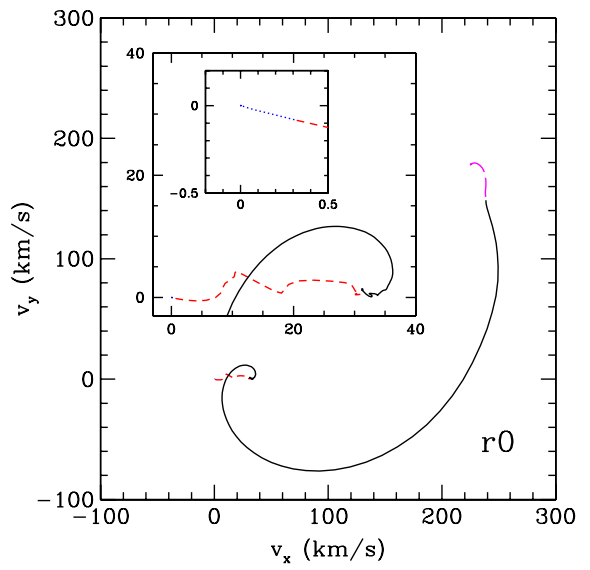

observer. The radiation received has information about the "correct" linear momentum of the spacetime which is solution of the Einstein equations for system $r 0$ as if it had inspiraled from infinity, and thus rapidly moves the center of mass to a net nonzero recoil velocity (cf., almoststraight dashed line in the inset in the left panel). Once the system has adjusted for the proper linear momentum, the evolution proceeds as expected, with the recoil velocity vector slowly tracking a spiral in velocity space. This is an important point which we prefer to underline: the rate of change of linear momentum is very large only initially and this is because as the binary migrates from the initial nonradiating state (the data is conformally flat) to the consistent radiating state, it will emit the amount of linear momentum it would have emitted when inspiralling from infinite separation. After this burst of linear momentum, the evolution of the recoil velocity is minute, essentially until it grows very rapidly during the last orbit.

Computing the integration constant consists then in calculating the position of the center of the spiral and this can be done either by a simple inspection of a graph in the velocity-space, from which compute the center of the spiral or, equivalently, by searching for the initial vector that would lead to an essentially monotonic in time growth of the recoil velocity [62]. The latter procedure does not require a human judgment but we have found it to yield the same answer (to less than $1 \mathrm{~km} / \mathrm{s}$ ) as the one guessed by looking at the velocity space.

The right panel of Fig. 6 shows the same evolution as the left one, but through different quantities. The upper panel, in particular, shows the time evolution of the recoil velocity

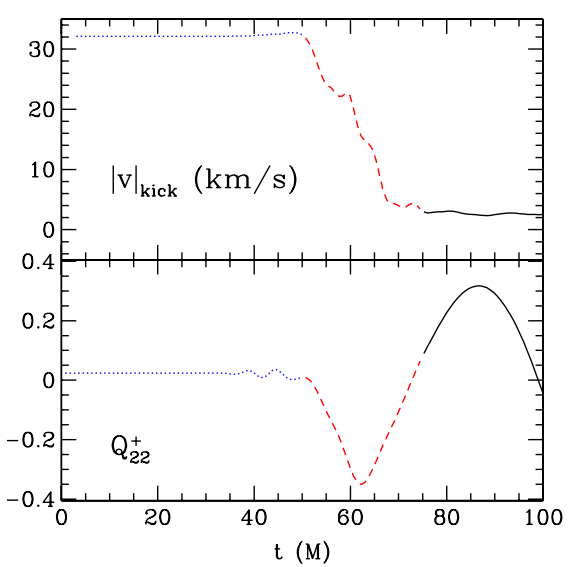

FIG. 6 (color online). Left panel: Evolution in velocity space of the recoil-velocity vector. Very little variation is recorded before the radiation reaches the observer at $r_{\mathrm{E}}=50 \mathrm{M}$ (dotted lines in the two insets). The absence of the proper linear momentum in the initial data triggers a rapid and an almost straight-line motion (dashed line) of the center of the spiral away from the origin of coordinates during the initial stages of the evolution. After this transient motion, the evolution is slower, with the spiral progressively opening up (solid line). The vector to the center of the spiral corresponds to the initial linear momentum of the spacetime and is used as integration constant for Eqs. (28) and (35). The final part of the evolution is characterized by a change in the spiral pattern (long-dashed line) as a result of the interaction of different modes in the ringdown of the final black hole. Note that the figure has been rotated clockwise of about $30^{\circ}$ to allow for the two insets. Right panel: Initial behavior of the recoil velocity (upper graph) and of the waveform $\left(Q_{22}^{+}\right)$for model $r 0$ (lower graph). This figure should be compared with the initial vector evolution of the recoil velocity shown in the left panel where the same types of lines have been used for the different stages of the evolution. 
and the rapid changes it undergoes initially when the radiation first invests the observer. The lower panel, on the other hand, shows the $Q_{22}^{+}$amplitude and highlights that, while the initial burst of radiation stops after $t \sim 50 \mathrm{M}$ (cf., dotted line), the waveform is still not fully consistent until $t \sim 75 M$ (cf., dashed line).

The procedure discussed so far for the calculation of the integration constant relative to the binary system $r 0$ applies qualitatively to all the other members of the sequence, with differences that are due essentially to the times at which the various stages take place.

It is worth remarking that the evolution of the recoil vector in the velocity-space has another interesting feature during the final stages of the evolution and when the final black hole is ringing down. This is marked as a longdashed line in the left panel of Fig. 6 and shows a break in the building of the spiral and the appearance of a new spiral with a different aperture (we refer to this feature as "the hook"). This is more evident in the left panel of Fig. 7, which shows the evolution of the recoil vector for the binary system $r 7$ and offers a magnification of the hook in the inset. A more detailed description of this feature is beyond the scope of this paper and will be presented in a future work, but we can here point out that the hook accounts for a rapid change in the recoil velocity and it is due to the interplay of different modes during the ringdown. This is clearly illustrated in the right panel of Fig. 7 which similarly reports the time evolution of the recoil velocity and the final stages of the $Q_{22}^{+}$waveform.

\section{B. Recoil velocities}

The recoil- velocity has been calculated for the sequence of models listed in Table I. As mentioned in Sec. II D, this sequence corresponds to equal-mass black holes, whose initial spins are unequal, though always aligned with the $z$-axis. The $r 0$ model has equal but opposite spins, while the $r 8$ model has equal and aligned spins on the black holes, with other models corresponding to intermediate values, as outlined in Sec. II D. Since the total initial orbital angular momentum $\boldsymbol{L}$ of the system is chosen to be constant over the sequence, the initial separations of the black holes increases in the sequence, as well as the time to merger due to spin-spin effects which contribute to an orbital "hang-up" in the aligned case.

We extract gravitational waves by both the gaugeinvariant and the $\Psi_{4}$ methods described in the previous section and by interpolating the radiation-related quantities onto 2-spheres at coordinate radii $r_{\mathrm{E}}=30 M, 40 M, 50 M$, and $60 \mathrm{M}$. The use of multiple extraction radii is made to check the consistency of the measurement and the precise value of the extraction radius has little influence on the actual kick calculation. In the case of the binary system $r 0$ we have verified that the recoil velocity yields the same value with differences that are smaller than $2 \mathrm{~km} / \mathrm{s}$ for extraction 2-spheres at distances larger than $30 \mathrm{M}$. As a result, we have used $r_{\mathrm{E}}=50 \mathrm{M}$ as the fiducial distance for an observer in the wave-zone and all of the results presented hereafter will be made at this extraction 2-sphere. A validation that the gauge-invariant quantities have the proper scaling with radius is presented in Appendix C.

The evolution of the recoil velocity for the entire sequence listed in Table I is displayed in the left panel of Fig. 8. It is apparent that the suitable choice of the integration constant discussed in the previous section yields early evolutions that are always monotonic in time and that, as expected, the largest recoil velocity is generated for the case in which the asymmetry is the largest, namely, for the binary $r 0$. The left panel Fig. 8 also shows that the
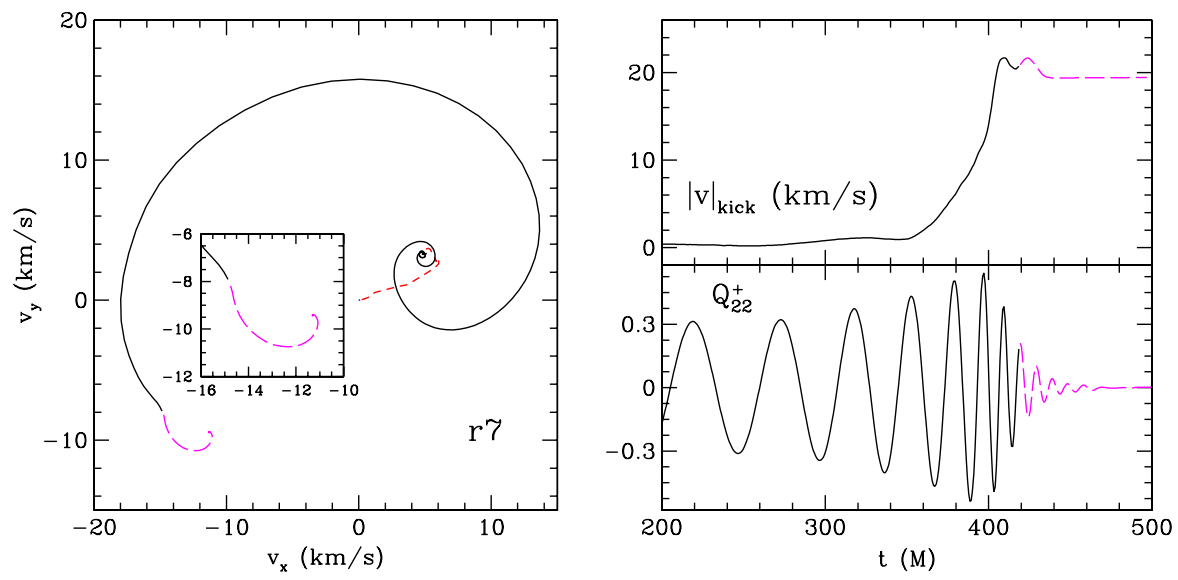

FIG. 7 (color online). Left panel: The same as in the left panel of Fig. 6 but for system $r 7$. Shown in the inset is the sudden reorientation of the recoil velocity vector during ringdown and corresponding to a new spiral with different aperture (long-dashed line). Although more pronounced in $r 7$, the appearance of this hook at ringdown is seen all the members of the sequence. Right panel: The same as in the left panel of Fig. 6 but for system $r$. The upper graph concentrates on the final stages of the evolution in of the recoil velocity and on the appearance of a second peak during ringdown (long-dashed curve). The lower graph shows the same but in terms of the $Q_{22}^{+}$waveform. A discussion of these final stages of the evolution is made in Sec. IV C. 

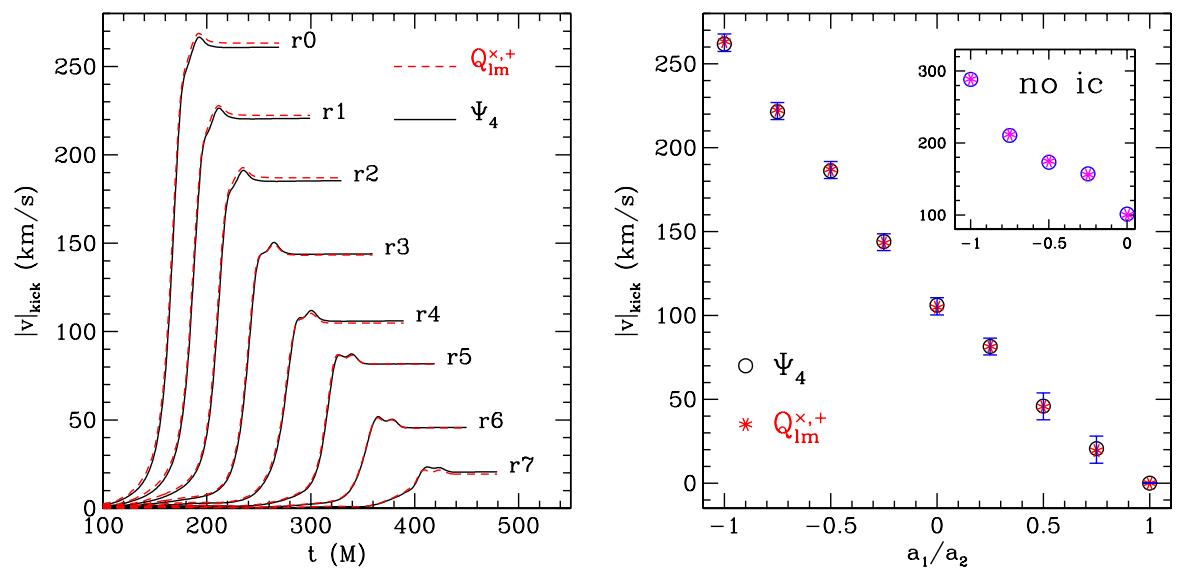

FIG. 8 (color online). Left panel: Recoil velocity as a function of the spin asymmetry parameter $a_{1} / a_{2}$ for the models listed in Table I. Indicated with a continuous lines are the results obtained via $\Psi_{4}$, while a dashed line is used for the gauge-invariant quantities $Q_{\ell m}^{+, \times}$. Right panel: Final recoil velocity calculated with both the use $\Psi_{4}$ (empty circles) and the gauge-invariant quantities (stars). Shown in the inset is the incorrect scaling obtained when the correction for the integration constant is not made.

profile for each case is rather similar, with the largest contribution to the kick velocity being generated in a period of about $80 \mathrm{M}$, corresponding roughly to the timescale of the last orbit and merger. Furthermore, it is notable that $95 \%$ of the acceleration occurs $\sim 30 M$ after the appearance of the first common apparent horizon, indicating that the kick is generated not only by the final stages of the inspiral (i.e., by the "plunge") but also and more significantly by the ringdown of the final black hole. This fact helps to explain why accurate recoil velocities can be obtained by evolutions involving very few cycles only, provided the integration constant is properly taken into account.

It is worth noting that during the final stages of the evolution, the recoil velocity is not monotonic but shows at least two peaks, whose relative amplitude depends on the spin ratio. For spin ratios $\sim-1$ the first peak is hardly visible, while the second one is the most pronounced one. As the spin ratio increases, however, the first peak becomes more prominent and for spin ratios $\sim 1$ it becomes comparable with the second one or even larger for binaries $r 6$ and $r 7$. As mentioned in the previous section and further discussed in the following one, the appearance of these peaks is related to the interplay of different mode-contributions during the ringdown. The second peak, in particular, can be associated to a rapid change in the recoil-velocity vector and is behind the characteristic "hook" discussed in the left panels of Figs. 6 and 7. While additional work is needed, especially in thorough perturbative investigations, to fully account for the rich, post-merger properties of the recoil velocities, we believe the double-peak evolution to be physically genuine since it is seen in all binaries and is supported by the highly accurate and convergent simulations. As a representative measure of the accuracy in determining these recoil velocities, we mention that we have carried out simulations also for the binary system $r 8$, in which the black holes have identical spin and thus from which no kick should result. The computed recoil velocity has been found to be $10^{-9} \mathrm{~km} / \mathrm{s}$, clearly indicating that our evolutions do an excellent job in preserving the orbital symmetry of these binaries.

We have found that the evolution of the recoil velocity generated by spin asymmetries appears to be rather different from the one generated by mass asymmetries $[8,10,47]$ and which shows much larger variations between the maximum attained value and the final one. Once again, this different behavior is related to the different interplay of the ringdown modes in the case of mass asymmetries and will be presented in a separate work.

The recoil velocities attained by the final black holes and shown for in the left panel of Fig. 8 can be studied in terms of their dependence on the spin ratio $a_{1} / a_{2}$, which can also be regarded as the "asymmetry" parameter of the system, being the largest for $a_{1} / a_{2}=-1$ and zero for $a_{1} / a_{2}=1$. These velocities are collected in Table II and are shown as a function of $a_{1} / a_{2}$ in the right panel of Fig. 8, where we have indicated with open circles the values obtained using $\Psi_{4}$ and with stars those obtained using the gauge-invariant perturbations.

The data in the right panel of Fig. 8 is shown together with its error-bars, which include errors from the determination of the integration constants, from the truncation error and from the amount of ellipticity contained in the initial data. We have estimated these errors to be of $5 \mathrm{~km} / \mathrm{s}$ for binaries $r 0-r 5$ and of $8 \mathrm{~km} / \mathrm{s}$ for binaries $r 6$ and $r 7$. Shown also in the inset is the recoil data obtained when ignoring the integration constant. It is remarkable that when the proper evaluation of the initial transient is not made, the data does not show the remarkable correlation with the spin ratio which is instead shown by the corrected data. Quite surprisingly, however, the correlation found the one predicted by PN studies. We recall, in fact, that using 
DENIS POLLNEY et al.

TABLE II. Final kick velocities in units of $\mathrm{km} / \mathrm{s}$ for the models listed in Table I. Columns two and three show the values obtained using the gauge-invariant quantities $Q_{\ell m}^{\times,+}$and $\Psi_{4}$ respectively and taking into account the integration constant. Columns four and five, on the other hand, show the results obtained when ignoring the integration constant. The same data are shown in the right panel of Fig. 8.

\begin{tabular}{lrrrr}
\hline \hline Model & $Q_{\ell m}^{\times,+}$ & $\Psi_{4}$ & $Q_{\ell m}^{\times,+}$, no ic & $\Psi_{4}$, no \\
\hline$r 0$ & 263.2 & 261.8 & 288.9 & 288.4 \\
$r 1$ & 222.4 & 221.4 & 211.9 & 210.6 \\
$r 2$ & 187.1 & 186.2 & 174.8 & 173.3 \\
$r 3$ & 143.3 & 144.0 & 155.9 & 157.3 \\
$r 4$ & 104.8 & 106.1 & 100.0 & 101.3 \\
$r 5$ & 81.4 & 81.5 & 76.9 & 77.0 \\
$r 6$ & 45.6 & 45.9 & 55.4 & 56.2 \\
$r 7$ & 19.4 & 20.6 & 13.8 & 14.8 \\
$r 8$ & 0.0 & 0.0 & 0.0 & 0.0 \\
\hline \hline
\end{tabular}

PN theory at the 2.5 order, Kidder [63] has concluded that in the case of a circular, nonprecessing orbit, the total kick for a binary system of arbitrary mass and spin ratio can be expressed as [5]

$$
\begin{aligned}
|v|_{\text {kick }} & =c_{1} \frac{q^{2}(1-q)}{(1+q)^{5}}+c_{2} \frac{a_{2} q^{2}\left(1-q a_{1} / a_{2}\right)}{(1+q)^{5}} \\
& =\tilde{c}_{2} a_{2}\left(1-\frac{a_{1}}{a_{2}}\right),
\end{aligned}
$$

where $q \equiv M_{1} / M_{2}$ is the mass ratio and is equal to one for the binaries considered here, thus leading to the second form of Eq. (40). The coefficients $c_{1}$ and $\tilde{c}_{2} \equiv c_{2} / 32$ depend on the total mass of the system and on the orbital separation at which the system stops radiating, which is intrinsically difficult to determine with precision since it lies in a region where the PN approximation is not very accurate. Indeed, we find that the coefficient $c_{2}$ is not really a constant in the case of equal-mass binaries but, rather, it can be seen to depend at least linearly on the spin ratio.

This is shown in Fig. 9, whose upper panel offers a comparison among the computed data for the recoil velocity (open circles) with the least-squares fits using either a linear (dotted line) or a quadratic dependence (dashed line). It is quite apparent that a linear dependence on $a_{1} / a_{2}$, such as the one expected in Eq. (40) for $c_{2}=$ const does not reproduce well the numerical data and yields pointwise residuals of the order of $20 \mathrm{~km} / \mathrm{s}$. These are shown with a dotted line in the lower panel of Fig. 9. A quadratic dependence on $a_{1} / a_{2}$, on the other hand, reproduces the numerical data very nicely, with residuals that are of the order of $5 \mathrm{~km} / \mathrm{s}$, as shown with a dashed line in the lower panel of the same figure, and thus compatible with the reported error-bars.
PHYSICAL REVIEW D 76, 124002 (2007)

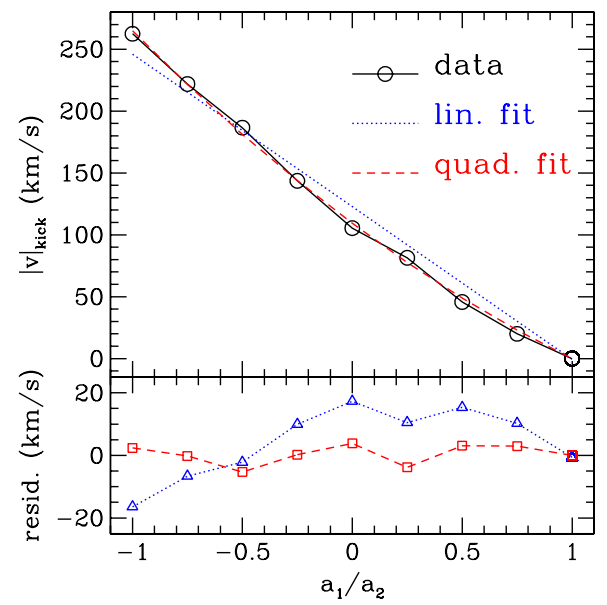

FIG. 9 (color online). Upper panel: Comparison of the computed data for the recoil velocity (open circles) with the leastsquares fits using either a linear (dotted line) or a quadratic dependence (dashed line). Lower panel: Point-wise residuals computed with the linear (dotted line) or a quadratic fit (dashed line).

We can reexpress Eq. (40) in the more generic form

$$
|v|_{\text {kick }}\left(a_{2}, \frac{a_{1}}{a_{2}}\right)=\left|a_{2}\right| f\left(\frac{a_{1}}{a_{2}}\right)
$$

where $a_{2}$ plays here the role of a "scale-factor". The function $f\left(a_{1} / a_{2}\right)$ with $a_{1} / a_{2} \in[-1,1]$ and maximum at $a_{1} / a_{2}=-1$ can then be seen as to be determined from numerical-relativity calculations (or higher-order PN approximations) and our least-squares fit suggests the expression

$$
f_{\text {quad }}=109.3-132.5\left(\frac{a_{1}}{a_{2}}\right)+23.1\left(\frac{a_{1}}{a_{2}}\right)^{2} \mathrm{~km} / \mathrm{s} .
$$

The maximum kick velocity for a given $a_{2}$ is then readily calculated even without a detailed knowledge of the function $f\left(a_{1} / a_{2}\right)$ as

$$
\left(|v|_{\text {kick }}\right)^{\max }\left(a_{2}\right)=\left|a_{2}\right| f(-1) .
$$

Using the data reported in Table II for $a_{2}=-0.584$ we obtain for $\left|a_{2}\right|=1$ that the maximum recoil velocity attainable from a binary system of equal-mass black holes with spins aligned to the orbital angular momentum is $448 \pm 5 \mathrm{~km} / \mathrm{s}$. This is in very good agreement with our previous estimate made in Ref. [10] with a smaller sequence and in equally good agreement with the results reported in Ref. [9].

\section{Mode contributions to the recoil velocity}

For the models studied in the previous section we have evaluated Eq. (35) including modes up to $\ell=7$. In practice, however, we find that the recoil is strongly determined by the lower-mode contributions. In particular, the two terms 


$$
\begin{gathered}
q_{1} \equiv \frac{1}{48 \pi} \sqrt{\frac{30}{7}} \dot{Q}_{22}^{+} Q_{3-3}^{+}, \\
q_{2} \equiv-\frac{\mathrm{i}}{48 \pi} \dot{Q}_{2-2}^{+} Q_{21}^{\times}
\end{gathered}
$$

are the dominant ones. This can be seen in Fig. 10, where the time evolutions of the terms $q_{1}$ and $q_{2}$ are plotted (dotted and dashed lines, respectively) together with the total kick calculated via Eq. (35) (solid line), and with the contributions from all other terms up to $\ell=7$ excluding $q_{1}$ and $q_{2}$ (long-dashed line). A rapid inspection of the figure reveals that the kick is dominated, in particular, by the $q_{2}$ term, whereas the $q_{1}$ term has a magnitude of the order of all the other modes combined. A similar result holds for each member of the sequence, so that the two contributions determine the final kick to more that $95 \%$. It should be noted that the mode contributions are vector quantities, just as the kick velocity itself, and are not always aligned or even maintain the same angle to each other during the duration of the recoil.

This coupling also goes some way to explain some features of the recoil velocity profiles displayed in Fig. 8. As mentioned in the previous section, in fact, the binaries $r 4$ to $r 8$ show a clear double peak in the evolution of the kick velocity before it settles down to the final value. The same feature can also be seen in the more asymmetric $r 0$ to $r 3$ binaries, where it appears as a flattening of the slope near the maximum. Since the two peaks are shown both by the gauge-invariant and by the $\Psi_{4}$-based techniques (which are rather different in both the assumptions they rely on and in the practical implementation) we do not believe them to be a simple numerical artifact. Overall, the properties of the recoil velocity near its maximum, and before it settles to the final value, are determined by the relative phases of the two contributions identified above.
An analysis of the terms $q_{1}$ and $q_{2}$ in vector-space, and which will be presented in a subsequent work, reveals that when they are relatively aligned at the peak of the acceleration, there is a clear single peak in the evolution. For the more symmetric models, on the other hand, the two contributions are more antialigned and a double peak results.

These considerations in the vector evolution of the two contributions $q_{1}$ and $q_{2}$ need also to be linked with the evolution in vector space of the recoil velocity. As stressed in Sec. IVA, in fact, there is a distinct kink in the evolution of the velocity vector towards the final stages of the merger (this feature is indicated with a long-dashed line in the $v_{x}$ vs $v_{y}$ plots of Figs. 6 and 7). The presence of the kink corresponds to a local decrease of the recoil velocity and hence to the minimum between the two peaks. Because this decrease is more pronounced for the lower-kick binaries $r 4$ to $r 8$, the first peak becomes more evident there.

\section{Angular momentum and mass conservation}

In this section we discuss the radiated angular momentum and energy during the evolution of the different initialdata sets. We compute the radiated angular momentum and mass by calculating their difference between the initial data and that of the final black hole, and then compare these quantities with the corresponding ones measured in terms of the emitted gravitational radiation. The differences in the two independent estimates serve therefore as stringent indicators of the conservative properties of our code.

The radiated angular momentum can be simply written as the difference between the initial and final values

$$
\boldsymbol{J}_{\mathrm{rad}}=\boldsymbol{J}_{\text {fin }}-\boldsymbol{J}_{\text {ini }}
$$

where, as a result of the conformal flatness of the initialdata slice, $\boldsymbol{J}_{\text {ini }}$ is given by the simple expression (see for

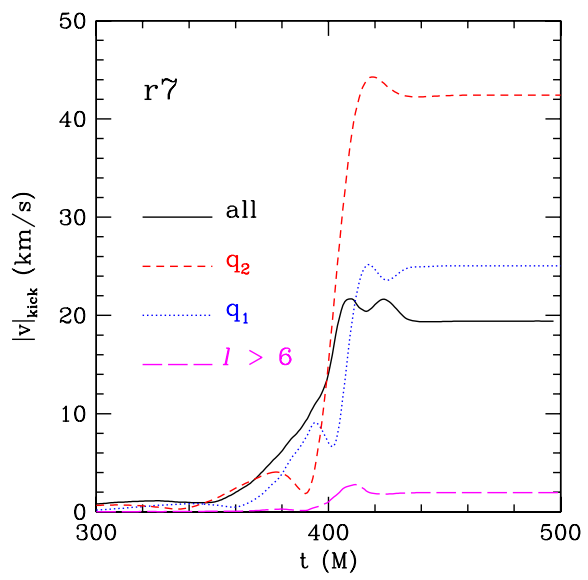

FIG. 10 (color online). The total kick calculated via Eq. (35) up to $\ell=7$ is compared to the contributions of individual terms $q_{1}$ and $q_{2}$, as well as the sum of term excluding these. In the case of the $r 0$ system (left panel) the spins are antialigned and the $q_{2}$ term is dominant and the $q_{1}$ term does not provide a significant contribution. In the case of the $r 7$ system (right panel), on the other hand, the spins are essentially aligned and the while the $q_{2}$ term is still dominant, the $q_{1}$ term also makes a significant contribution. 
example [39-41] and discussion in Sec. II)

$$
J_{\mathrm{ini}} \equiv J_{\mathrm{ADM}}=\boldsymbol{C}_{1} \times p_{1}+C_{2} \times p_{2}+S_{1}+S_{2} .
$$

Here $\boldsymbol{C}_{i}, \boldsymbol{p}_{i}$ and $\boldsymbol{S}_{i}$ are the position, the linear momentum and the spin of the $i$ th black hole. The final angular momentum $\boldsymbol{J}_{\text {fin }}$, on the other hand, is set to be equal to the spin of the final black hole after all the radiation has left the computational domain. Two different methods are used to obtain this measure, both of which are based on properties of the apparent horizon of the final hole.

The first method employs the isolated/dynamical horizon formalism and searches for a rotational Killing vector $\phi^{a}$ on the final apparent horizon so as to measure the spin of the final black hole as [64-66]

$$
J=-\frac{1}{8 \pi} \oint_{S} K_{a b} \phi^{a} \hat{r}^{b} d^{2} V .
$$

We note that this expression (48) is valid on any sphere where a Killing vector $\phi^{a}$ can be found, and is therefore a quasilocal measure of the angular momentum. In particular, at large distances where the spacetime is close to axisymmetric, there is a good approximation to an angular Killing vector, and we can apply this expression to determine the angular momentum of the spacetime. Note also that Eq. (48) is identical to the ADM angular momentum when evaluated at spacelike infinity. (Refs. $[65,66]$ also give a quasilocal formula for the angular momentum flux due to gravitational radiation.)

The second method instead, assumes that the final black hole has settled to a Kerr one and uses the rotationalinduced distortion of the apparent horizon of the final black hole to estimate its spin. Defining $C_{p}$ and $C_{e}$ to be, respectively, the apparent horizon's polar and equatorial proper circumferences, their ratio $C_{r} \equiv C_{p} / C_{e}$ will undergo damped oscillations as the perturbed black hole settles to a Kerr state through the quasinormal ringing. The final value of $C_{r}$ can be expressed as a nonlinear function of the dimensionless spin parameter $a=J / M^{2}$ as [21,6769]

$$
C_{r}(a)=\frac{1+\sqrt{1-a^{2}}}{\pi} E\left(-\frac{a^{2}}{\left(1+\sqrt{1-a^{2}}\right)^{2}}\right),
$$

where $E(k)$ is the complete elliptic integral of the second kind

$$
E(k)=\int_{0}^{\pi / 2} \sqrt{1-k \sin ^{2} \theta} d \theta .
$$

By inverting numerically Eq. (49) we obtain $a$ from the late time $C_{r}$ that is measured from the apparent horizon shape. Note that for computing $J$ we need to multiply $a$ by the square of the final mass, which we take to be $M_{\mathrm{ADM}}-$ $M_{\text {rad }}$. An alternative choice involving the total mass equation (17) as measured from the apparent horizon would lead to essentially the same results.
As mentioned at the beginning of this section, the determination of the radiated angular momentum can also be done using directly the asymptotic waveform amplitudes $h_{+}$and $h_{\times}$as $[55,70,71]$

$$
\frac{d^{2} J}{d t d \Omega}=-\frac{r^{2}}{16 \pi}\left(\partial_{t} h_{+} \partial_{\phi} h_{+}^{*}+\partial_{t} h_{\times} \partial_{\phi} h_{\times}^{*}\right),
$$

where the amplitude $h_{+}$and $h_{\times}$themselves can be expressed either in terms of the Zerilli-Moncrief gaugeinvariant variables $Q_{\ell m}^{+}, Q_{\ell m}^{\times}$or, alternatively, in terms of the Newman-Penrose scalar $\Psi_{4}$. A comparison between the two approaches is presented in Appendix C, where it is shown that the differences are minute. Because of this, hereafter we will refer to asymptotic amplitudes measured in terms of the gauge-invariant variables only. Additional details on the resolution of the extraction 2-sphere are also presented in Appendix B.

The left panel of Fig. 11 summarizes this comparison by showing, as functions of the spin ratio $a_{1} / a_{2}, \mathrm{M} J_{\text {fin }}$ from Eq. (48), $J_{\text {rad }}$ from Eq. (51) both adding nicely to yield $J_{\text {ini }}$. Note that $J_{\text {ini }}$ is growing linearly as it is obvious from Eq. (47), but also that that a similar behavior is shown by the radiated angular momentum (and hence by the final spin of the black hole). Using a linear fitting we can derive phenomenological expressions for the relative losses of angular momentum

$$
\frac{J_{\mathrm{rad}}}{J_{\mathrm{ini}}}=\xi_{\mathrm{rad}}^{J}\left(\frac{a_{1}}{a_{2}}\right)+\chi_{\mathrm{rad}}^{J},
$$

and the relative spin-up of the final black hole

$$
\frac{J_{\text {fin }}}{J_{\text {ini }}}=\xi_{\text {fin }}^{J}\left(\frac{a_{1}}{a_{2}}\right)+\chi_{\text {fin }}^{J} .
$$

The fitted values for $\xi_{\text {rad,fin }}^{J}$ and $\chi_{\text {rad,fin }}^{J}$ are presented in Table III and readily indicate that the system loses $24 \%$ of its initial orbital angular momentum in the case of antialigned spins and up to $34 \%$ for aligned spins.

To the best of our knowledge expressions (52) and (53) do not have a PN counterpart and yet, since they depend only on the spin-ratio, they represent simple and powerful ways of estimating both the efficiency in the extraction of angular momentum and the spin of the final black in a binary merger when the spins are orthogonal to the orbital plane. This information could be easily injected in those $N$-body simulations in which the interaction of binary black holes is taken into account [72] and thus yield accurate estimates on final distribution of black-hole spins.

Since we have two independent and different ways of computing $J_{\text {rad }}$ [i.e., either from Eq. (51) or from Eq. (46)] we can quantify our ability to conserve angular momentum by measuring the normalized residual

$$
\frac{\Delta J}{J_{\text {ini }}} \equiv \frac{J_{\text {fin }}+J_{\text {rad }}-J_{\text {ini }}}{J_{\text {ini }}} .
$$

This is shown in the right panel of Fig. 11 and the two 

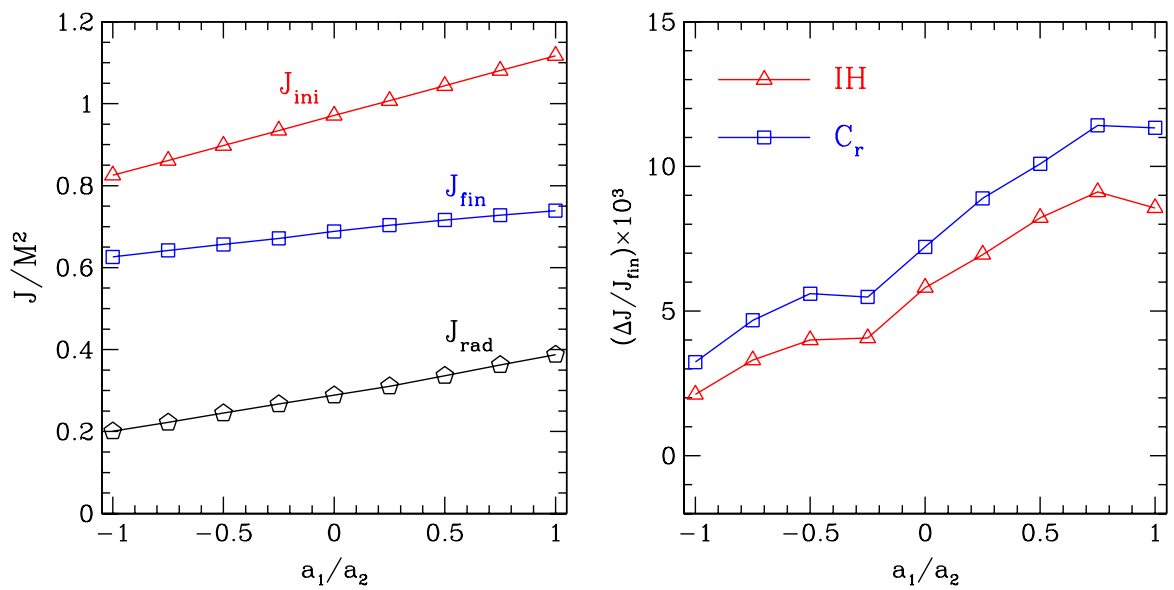

FIG. 11 (color online). Left panel: Dependence on the spin ratio of the initial total angular momentum $J_{\text {ini }}$ [as computed from Eq. (47)], of the radiated angular momentum $J_{\text {rad }}$ [as computed through the gauge-invariant waveforms], and of the final spin of the black hole $J_{\text {fin }}$. All quantities show a linear behavior, whose coefficient are collected in Table III. Right panel: Relative error $\Delta J / J_{\text {ini }}$ in the conservation of the angular momentum [cf., Eq. (54)]. Different curves refer to whether the final spin of the black hole is computed using the isolated/dynamical horizon formalism (triangles) or the distortion of the apparent horizon (squares). In both cases the error is of about $1 \%$ at most for simulations at the medium resolution.

different lines refer to the two measures of the final spin of the black hole, i.e., either via the isolated-horizon formalism (triangles) or via the distortion of the apparent horizon (squares). In both cases the error is extremely small, ranging between $1.1 \%$ and $0.2 \%$ for simulations at the medium resolution, and thus providing convincing evidence of our accuracy in the preservation of angular momentum. It should be noted that while there seems to be a small advantage in using the isolated horizon measure, the differences are too small to be significant. Indeed, a small change in the procedure, such as the use of the mass measured via the apparent horizon via Eq. (49) in place of $M_{\text {ini }}-M_{\text {fin }}$ (as we are doing in this figure), would revert the advantage.

We proceed next to a similar analysis for the conservation of the mass-energy of the system by considering the difference between the initial mass and final plus the radiated masses. As for the initial mass we obviously consider the ADM mass of the system $M_{\mathrm{ADM}}$, while the radiated energy $M_{\text {rad }}$ is computed through the gravitational waveforms $[55,73,74]$

TABLE III. Coefficients for the phenomenological expressions (52) and (53) (and the corresponding coefficients for $\left.\Delta M_{\text {rad,fin }} / M\right)$ by means of which it is possible to compute the relative losses of energy and angular momentum, as well as the final mass and spin of the black hole in binary mergers in which the spins are orthogonal to the orbital plane.

\begin{tabular}{lrcr}
\hline \hline$\xi_{\text {rad }}^{J}$ & 0.0513 & $\xi_{\text {rad }}^{M}$ & 0.0118 \\
$\chi_{\text {rad }}^{J}$ & 0.2967 & $\chi_{\text {rad }}^{M}$ & 0.0437 \\
$\xi_{\text {fin }}^{J}$ & -0.0513 & $\xi_{\text {fin }}^{M}$ & -0.0118 \\
$\chi_{\text {fin }}^{J}$ & 0.7033 & $\chi_{\text {fin }}^{M}$ & 0.9563 \\
\hline
\end{tabular}

$$
\frac{d^{2} E}{d t d \Omega}=\frac{r^{2}}{16 \pi}\left(\left|\dot{h}_{+}\right|^{2}+\left|\dot{h}_{\times}\right|^{2}\right)
$$

As for the angular momenta, we have chosen to express the right-hand side of Eq. (55) in terms of the Zerilli-Moncrief functions and to use as final mass of the black hole $M_{\text {fin }}$, the one given by Eq. (17) and measured via the apparent horizon.

The left panel of Fig. 12 shows $M_{\mathrm{ADM}}, M_{\mathrm{fin}}$ and $M_{\mathrm{rad}}$, with the latter rescaled the radiated by a factor of 10 to make it more visible. Also in this case there is a clear linear behavior of both the radiated energy and of the final mass of the black hole in terms of the spin ratio. As a result, phenomenological expressions of the type (52) and (53) are possible also for $M_{\text {fin }}$ and $M_{\text {rad }}$. The corresponding values of the coefficients $\xi_{\text {rad,fin }}^{M}$ and $\chi_{\text {rad,fin }}^{M}$ are also presented in Table III.

Finally, to check the precision at which the energy is conserved, and in analogy to Eq. (54), we have computed the relative error

$$
\frac{\Delta M}{M_{\mathrm{ADM}}} \equiv \frac{M_{\mathrm{fin}}+M_{\mathrm{rad}}-M_{\mathrm{ADM}}}{M_{\mathrm{ADM}}},
$$

and plotted this as a function of the spin ratio in the right panel of Fig. 12. Clearly, also the energy losses are extremely small and for all the binaries in the sequence, the error in the energy balance is below $0.52 \%$ at the medium resolution. Table IV summarizes the numerical results for the radiated energy and angular momentum for the members of the sequence. 

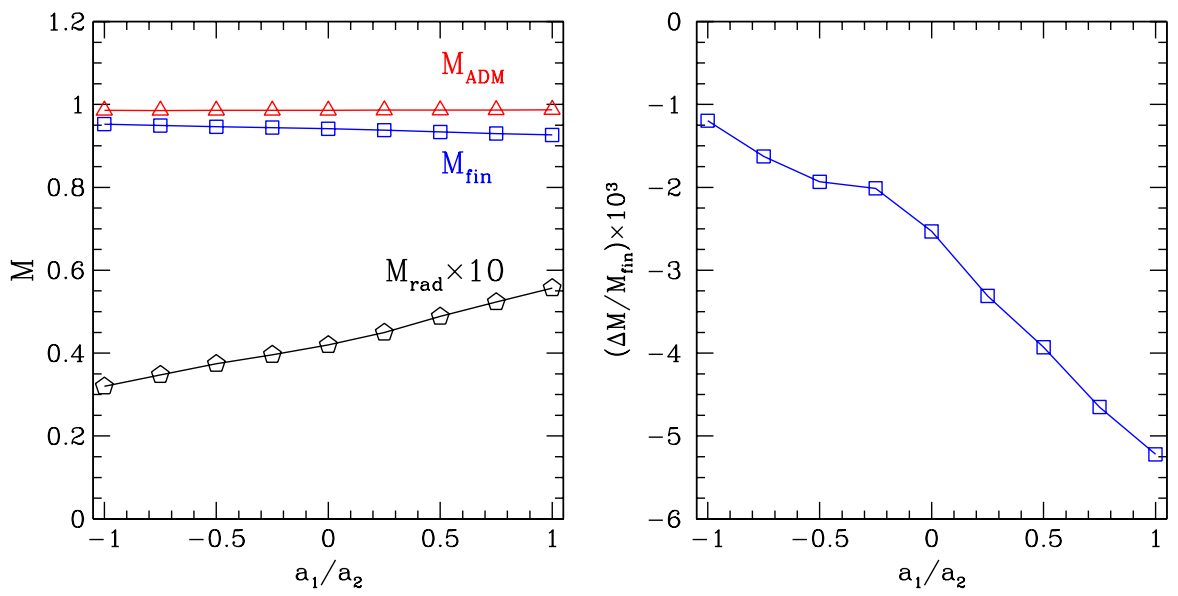

FIG. 12 (color online). Left panel: Dependence on the spin ratio of the ADM mass $M_{\mathrm{ADM}}$, of the scaled radiated energy $M_{\text {rad }}$ [as computed through the gauge-invariant waveforms and scaled by a factor of 10 to make it visible], and of the final mass of the black hole $M_{\text {fin }}$. All quantities show linear behaviors, whose coefficients are collected in Table III. Right panel: Relative error $\Delta M / M_{\text {ini }}$ in the conservation of the energy [cf., Eq. (56)]. Note that the error is of about $0.5 \%$ at most for simulations at the medium resolution.

TABLE IV. Final and radiated angular momenta and masses, computed from the gauge-invariant waveforms. Shown is also the radiated spin and mass relative to their initial values, which are listed in Table I.

\begin{tabular}{rrccccccc}
\hline \hline \multicolumn{2}{r}{$a_{1} / a_{2}$} & $J_{\text {fin }}$ & $J_{\text {rad }}$ & $J_{\text {rad }} / J_{\mathrm{ADM}}$ & $M_{\text {fin }}$ & $M_{\text {rad }}$ & $M_{\text {rad }} / M_{\mathrm{ADM}}$ \\
\hline$r 0$ & -1.00 & 0.6244 & 0.2008 & 0.2434 & 0.9536 & 0.0320 & 0.0325 \\
$r 1$ & -0.75 & 0.6391 & 0.2222 & 0.2580 & 0.9507 & 0.0348 & 0.0353 \\
$r 2$ & -0.50 & 0.6530 & 0.2449 & 0.2727 & 0.9482 & 0.0374 & 0.0380 \\
$r 3$ & -0.25 & 0.6676 & 0.2670 & 0.2857 & 0.9461 & 0.0396 & 0.0402 \\
$r 4$ & 0.00 & 0.6827 & 0.2886 & 0.2971 & 0.9439 & 0.0420 & 0.0426 \\
$r 5$ & 0.25 & 0.6966 & 0.3106 & 0.3084 & 0.9412 & 0.0450 & 0.0456 \\
$r 6$ & 0.50 & 0.7075 & 0.3363 & 0.3222 & 0.9376 & 0.0488 & 0.0495 \\
$r 7$ & 0.75 & 0.7181 & 0.3626 & 0.3355 & 0.9344 & 0.0523 & 0.0530 \\
$r 8$ & 1.00 & 0.7292 & 0.3878 & 0.3471 & 0.9315 & 0.0557 & 0.0564 \\
\hline \hline
\end{tabular}

\section{CONCLUSIONS}

We have performed a highly-accurate study of recoil velocities in binary black hole mergers from a sequence of equal-mass black holes with varying spin configurations. In this sequence, the spins are aligned with the orbital angular momentum since there are strong indications that such alignment is preferred in astrophysical situations. This makes our choice of initial data especially realistic and our results particularly relevant also within an astrophysical context.

In practice, the initial configurations are built so that the spin of one of the black holes is kept at a constant dimensionless value $a_{2}=0.584$ while the other varies from $a_{1}=$ $-a_{2}$ to $a_{1}=+a_{2}$, thus spanning a range between -1 and 1 in spin ratio. We have followed our black hole evolutions for about two to four orbits and then throughout the plunge, merger, and ringdown phases. This work thus extends and refines recent results obtained from a reduced but similar initial-data sequence [10].
The main aspects of this work, which revolve around the methods used, the tests performed and the results obtained, can be summarized as follows.

Methods. To increase the significance of our results and our confidence in their accuracy, we have implemented two independent methods for the calculation of the linear momentum from the emitted gravitational radiation. These are based on either the measure the Newman-Penrose scalar $\Psi_{4}$ or on the calculation of the gauge-invariant perturbations of a Schwarzschild black hole $Q_{\ell m}^{\times,+}$. Overall, we find that both methods of calculating the linear momentum loss agree excellently and we are thus able to obtain accurate recoil measurements with error bars of $5 \mathrm{~km} / \mathrm{s}$ for the antialigned spin binaries and of $8 \mathrm{~km} / \mathrm{s}$ in the aligned cases.

Such a good agreement, however, is attainable only if the initial transient in the waveform is properly taken into account. The transient is produced by the use of initial data not containing the net linear momentum the system has accumulated since inspiralling from infinite separation. We discuss the importance of choosing the correct vector integration constant when calculating the radiated linear momentum and describe an unambiguous method for doing so.

We remark that a proper choice of this constant is essential not only because it influences the final recoil velocity with differences of $10 \%$ and more, but also because it allows for a systematic interpretation of the results. Without it, in fact, the correct functional dependence of the final recoil velocity on the spin ratio is irremediably lost and a comparison with the PN prediction impossible. Last but not least, a proper integration constant can result in a significant saving of computational time, allowing simulations to start at much smaller initial separations without sacrificing accuracy.

Tests. In order to show the accuracy of our results, we demonstrate that both the Zerilli-Moncrief gauge invariant 
waveforms and the Einstein tensor converge with an order between three and four, which is the expected convergence behavior of our numerical methods.

Furthermore, because the Newman-Penrose scalar $\Psi_{4}$ serves as a measure for the radiation content of the spacetime in appropriately chosen frames and at sufficiently large distances from the source, we show that the peeling property is indeed well satisfied in our numerical simulations. In particular, we demonstrate that both the gravitational wave information $\Psi_{4}$ and the gauge-wave information $\Psi_{3}$ satisfy the expected scaling with radius. Similarly, we also show that, as expected, the gauge invariant quantity $Q_{22}^{+}$does not vary with radius.

Finally, we investigate those systematic effects that may influence our gravitational-wave measurements. In particular, we study the effects that the choice of the extraction radius has on the final kick velocity and find little influence for $r_{\mathrm{E}} \geq 30 \mathrm{M}$. Based on this, we choose $r_{\mathrm{E}}=50 \mathrm{M}$ as the fiducial extraction radius in this paper. Furthermore, to exclude that the effects of the eccentricity in our initial data are significant for this paper, we artificially increase or reduce the eccentricity of the initial data by comparatively large amounts. Also in this case we find that the differences in the recoil velocities are below the estimated error-bars. Altogether, the set of tests carried out gives us confidence that our waveforms and recoil velocities are both correct and accurate.

Results. Using the mathematical and numerical setup as described and tested above, we have investigate the dependence of the recoil velocity on the initial data parameters and most notably on the spin ratio $a_{1} / a_{2}$. As expected, a larger asymmetry in the initial conditions causes a larger recoil, with a velocity of about $262 \mathrm{~km} / \mathrm{s}$ for a binary of equal and antialigned spins, and a numerically computed recoil of $10^{-9} \mathrm{~km} / \mathrm{s}$ for a binary of equal and aligned spins.

Using such accurate measurements, we have then studied the functional dependence of the recoil velocity on the spin ratio finding that a quadratic behavior reproduces very well the numerical results and corrects the postNewtonian prediction of a linear dependence. We summarize this behavior in a phenomenological expression that can be readily employed in astrophysical studies on the evolution of binary black holes in massive galaxies.

With a straightforward extrapolation of the quadratic dependence to the maximal spinning case $a_{1}=-a_{2}=1$ we obtain $448 \pm 5 \mathrm{~km} / \mathrm{s}$ as the maximal possible recoil velocity attainable from a binary system of equal-mass black holes with spins aligned to the orbital angular momentum. This recoil velocity is in very good agreement with our previous estimate made in Ref. [10] with a smaller sequence and in equally good agreement with the results reported in Ref. [9].

As mentioned above, the inclusion of the integration constant has been essential to obtain physically consistent results. At the same time, its investigation has allowed to highlight some important features of the evolution of the recoil velocity in vector space. Most importantly, it has shown that even when all nonspherical modes up to $\ell=7$ are taken into account, the recoil is dominated by lower mode contributions, especially $\ell=2, m=-2,1,2$ and $\ell=3, m=-3$. The interplay of these contributions in vector space and during ringdown is what is responsible for the rich features observed in the final evolution of the recoil velocity.

Finally, we provide accurate measurements of the radiated energy and angular momentum. These measurements reveal a clear linear dependence on the spin ratio $a_{2} / a_{1}$, and we derive phenomenological expressions for the relative losses of angular momentum and the relative spin-up of the final black hole. These relations can be easily used in $N$-body simulations if the interaction of binary black holes is to be taken into account, and when an accurate estimate on the final distribution of black hole spins is important.

\section{ACKNOWLEDGMENTS}

It is a pleasure to thank Thibault Damour for interesting discussions. A. N. and E. S. also acknowledge kind hospitality from the AEI during the development of part of this work. The computations were performed on the clusters Peyote, Belladonna, and Damiana of the Albert Einstein Institute. This work was supported in part by the DFG grant SFB/Transregio 7 "Gravitational Wave Astronomy".

\section{APPENDIX A: ON THE CONVERGENCE TESTS}

The effects of the initial transient modes can last for different amounts of time for the different resolutions. A comparison of the $Q_{22}^{+}$waveforms between the three resolutions confirms this shift in time- the waveform maxima are seen at slightly different times for the different resolutions. We attempt to undo this effect by manually shifting the time-coordinate of the medium and high resolution runs

$$
t \rightarrow t+\delta t
$$

The value of $\delta t$ is set for the medium and high resolution runs independently, using the minimization condition

$$
\frac{\partial}{\partial(\delta t)} \int_{150}^{170}\left|Q(t \rightarrow t+\delta t)-Q_{\text {vhigh }}\right|^{2} d t=0 .
$$

This effectively means aligning in time the peak amplitude of the three runs, at $t \approx 160 M$. Solving Eq. (A2) numerically for the $Q_{22}^{+}$waveforms gives

$$
\delta t_{0.024}=0.4756 \text { and } \delta t_{0.018}=0.1078 .
$$

Applying the time-shifting condition Eq. (A1) to the coarse and medium resolution data, and inserting the result into Eqs. (20) and (21) gives convergence rates that are consistent with the theoretical expectations. 
TABLE V. Integrated convergence rates of the ZerilliMoncrief gauge-invariant variables providing the dominant contribution in the kick-velocity measurements. As the numbers indicate, we achieve at least third order convergence both in amplitude and phase. A time-shift as given by Eqs. (A1)-(A3) was made on the raw data to remove the near cancellation of the lowest-order error terms.

\begin{tabular}{lcccccc}
\hline \hline$Q$ & \multicolumn{2}{c}{$Q_{21}^{\times}$} & \multicolumn{2}{c}{$Q_{22}^{+}$} & \multicolumn{2}{c}{$Q_{33}^{+}$} \\
$r_{\mathrm{E}} / M$ & amp & phase & amp & phase & amp & phase \\
\hline 30 & 4.51 & 3.95 & 4.65 & 4.31 & 4.32 & 2.13 \\
40 & 4.08 & 3.70 & 4.61 & 4.34 & 4.26 & 2.62 \\
50 & 3.83 & 4.44 & 4.35 & 4.76 & 4.02 & 2.39 \\
\hline \hline
\end{tabular}

In Table $\mathrm{V}$ we report the convergence rates as calculated from Eq. (20) for the time interval $0 \leq u \leq 190(u$ is the retarded time as defined in Sec. IIE) which excludes the initial burst but contains the rest of the waveform. We see close to fourth-order convergence for the $\ell=2$ modes $Q_{22}^{+}$ and $Q_{21}^{\times}$. The $\ell=m=3$ mode $Q_{33}^{+}$, on the other hand, shows second order convergence in phase, which is most likely related to the fact that the magnitude of this mode is the same size as the finite difference error in $Q_{22}^{+}$and is a factor of 40 smaller than the magnitude of $Q_{22}^{+}$itself.

The final kick-velocity magnitude in units of $\mathrm{km} / \mathrm{s}$ is

$$
|v|_{\text {kick }}=263.49, \quad 259.75, \quad \text { and } 261.00
$$

for the medium, high and very-high resolutions. This gives $\rho\left(|v|_{\text {kick }}\right)=2.98$ which can be inserted into Eq. (21) to obtain a calculated convergence rate of 4.32 .

\section{APPENDIX B: DETAILS ON THE EXTRACTION $\mathrm{OF} \Psi_{4}$}

The numerical solution of Eqs. (28) involves first an interpolation of $\Psi_{4}$ as calculated according to Eqs. (24) from its values on the Cartesian grid to those onto the extraction sphere by using fourth-order Lagrange interpolants. Because of the symmetry across the $z=0$ plane the interpolation is effectively done on the upper hemisphere only, thus using a spherical coordinate system with $\theta, \phi \in$ $[0, \pi / 2] \times[0,2 \pi]$ and applying cell-centered discretization along the $\theta$-direction to avoid the coordinate singularities at the poles on the sphere.

The angular resolution is chosen so that the spacings $\Delta \theta$ and $\Delta \phi$ are equal and of the same order as the corresponding Cartesian spacings of the refinement level in which the largest extraction 2-sphere is located. As an example, for the fiducial finest resolution of $h=0.024 M$, the largest extraction radius is at $r_{\mathrm{E}}=60 \mathrm{M}$ and in a region covered by the second refinement level with spacing $\Delta_{r l=2}^{0.024}=1.536 \mathrm{M}$. To obtain an equivalent spacing on the 2 -sphere, we solve for $\Delta \theta$ and $\Delta \phi$ such that

$$
r_{\mathrm{E}} \Delta \theta=r_{\mathrm{E}} \Delta \phi \approx \Delta_{r l=2}^{0.024}=1.536 M .
$$

The resulting number of grid points is $N_{\theta}=56$ along the $\theta$-direction and $N_{\phi}=224$ along the $\phi$-direction.

After interpolation onto the extraction sphere, we first calculate the time integral of $\left.\Psi_{4}\right|_{S^{2}}$ and afterwards, the surface integral of the absolute square of the former according to Eqs. (28). These integrals are both computed using fourth-order schemes. In particular, for the surface integral, we use Simpson's rule in the form

$$
\begin{aligned}
\int_{x_{0}}^{x_{N}} d x f(x) \approx & \Delta x\left[\frac{17}{48} f_{0}+\frac{59}{48} f_{1}+\frac{43}{48} f_{2}+\frac{49}{48} f_{3}\right. \\
& +\left\langle f_{k}\right\rangle+\frac{49}{48} f_{N-3}+\frac{43}{48} f_{N-2}+\frac{59}{48} f_{N-1} \\
& \left.+\frac{17}{48} f_{N}\right]
\end{aligned}
$$

where $\left\langle f_{k}\right\rangle$ is the sum over all $f_{k}$ with $3<k<N-3$. The integral over $d \theta d \phi$ is obtained by computing the tensor product of the RHS of Eqs. (B2), i.e.,

$$
\int_{\theta_{0}}^{\theta_{N}} d \theta \int_{\phi_{0}}^{\phi_{N}} d \phi f(\theta, \phi) \approx \Delta \theta \Delta \phi \sum_{i=0}^{N_{\theta}} \sum_{j=0}^{N_{\phi}} c_{i} c_{j} f_{i j},
$$

where the $c_{i}, c_{j}$ are the coefficients in the RHS of Eqs. (B2).

The time integral of Eqs. (28) is generically calculated by using the fourth-order Simpson's rule in such a way that the integral for the time step $k$ uses only past time steps $i$ with $0 \leq i \leq k$. Care is required for the very first time steps, for which we have less than 7 evaluations of the integrand. In this case, we use the 2nd-order accurate trapezoid rule if $N=1,3$, or 5

$$
\int_{x_{0}}^{x_{N}} d x f(x) \approx \Delta x\left[\frac{1}{2} f_{0}+\left\langle f_{k}\right\rangle+\frac{1}{2} f_{N}\right],
$$

or the fourth-order accurate Simpson's rule

$$
\begin{aligned}
\int_{x_{0}}^{x_{N}} d x f(x) \approx & \Delta x\left[\frac{1}{3} f_{0}+\frac{4}{3} f_{1}+\left\langle\frac{2}{3} f_{2 k}+\frac{4}{3} f_{2 k+1}\right\rangle\right. \\
& \left.+\frac{1}{3} f_{N}\right],
\end{aligned}
$$

if $N=2,4$ or 6 . For $N \geq 7$ we simply use Simpson's rule in the form (B2). It should be noted that the use of a higherorder time integration scheme improves the overall accuracy in the calculation of the final recoil velocity by more than a factor of 10 .

\section{APPENDIX C: A COMPARISON OF WAVE- EXTRACTION METHODS}

In Fig. 4, we have shown that $\Psi_{4}$ as extracted at different radii correctly scales with the $1 / r$ falloff as predicted by the peeling theorem. Here, we also check if all other components of the Weyl tensor exhibit the correct $r^{5-n} \Psi_{n}=$ const scaling. 
The left panel of Fig. 13 indeed shows that the scaling property of all $\Psi_{n}$ behave as expected. In the course of the same analysis, it is also worth looking at the waveforms as calculated by using the gauge-invariant formalism. In particular, we focus on the real part of the $\ell=2, m=2$ even parity wave mode $Q_{22}^{+}$and check for the correct scaling for the different extraction radii. The right panel of Fig. 13 shows that $Q_{22}^{+}$is constant for all extraction radii as expected.

As a final remark, we will also compare the $h_{+}$and $h_{\times}$as calculated by using the odd and even master functions in the gauge-invariant formalism according to Eq. (30) and the spin-weighted spherical harmonic amplitudes of the Weyl component $\Psi_{4}^{\ell m}$ decomposed on the extraction spheres. Using these amplitudes, the metric perturbations $h_{+}, h_{\times}$recovered by a double time integral of Eq. (26)
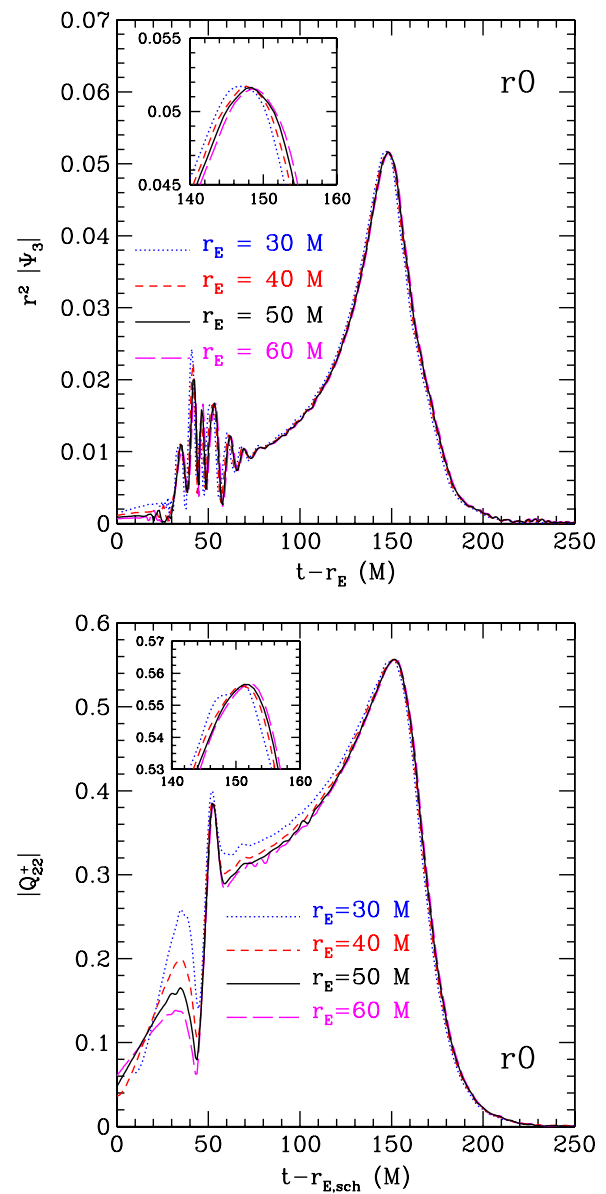

FIG. 13 (color online). Left panel: Evidence that the conditions for the Peeling theorem are met also for $\Psi_{3}$, which scales as $r^{-2}$ when extracted at isotropic radii $r_{\mathrm{E}}=30 \mathrm{M}, 40 \mathrm{M}, 50 \mathrm{M}$, and $60 \mathrm{M}$. This figure should be compared to the corresponding Fig. 4. Right panel: The same as the left panel but for the gaugeinvariant quantity $Q_{22}^{+}$, which is shown to be constant when extracted at isotropic radii $r_{\mathrm{E}}=30 M, 40 M, 50 M$, and $60 M$.

$$
h_{+}-\mathrm{i} h_{\times}=\lim _{r \rightarrow \infty} \sum_{\ell, m} \int_{0}^{t} d t^{\prime} \int_{0}^{t^{\prime}} d t^{\prime \prime} \Psi_{4}^{\ell m}{ }_{-2} Y_{\ell m} .
$$

The numerical integration of Eq. (C1) requires knowledge of an integration constant for the calculation of the second integral to eliminate the linear offset. This constant is determined by searching for minima in the $\Psi_{4}^{\ell m}$ mode and averaging over them. The resulting value is used as the integration constant. In both cases, we only consider the dominant contribution from mode $\ell=2, m=2$. In Figure 14, we show a comparison between waves extracted via $\Psi_{4}^{l m}$ and the gauge invariant $Q_{l m}^{+}$.

\section{APPENDIX D: ON THE INFLUENCE OF ORBITAL ECCENTRICITY}

Another source of potential error in calculating a "physical" kick comes from the choice of initial data parameters. Our evolutions begin from fairly close separations, comprising at most the last $2-3$ orbits. As such, parameters for quasicircular orbits determined by the effective potential method, give only approximations to the true orbital parameters for black holes that have spiraled in from infinity, and it is known that the method produces a nontrivial residual eccentricity for initial data at close separation. This eccentricity can have significant effects on the orbital trajectories before merger, and a potential influence on the calculated recoil. To test this we have evolved two modified $r 0$ models, one in which the initial linear momenta of the black holes is 3\% larger than that specified in Table I, and another in which the linear momenta are $3 \%$ smaller. The modified momenta have the effect of changing the orbital energy of the bodies from the minima determined by the effective potential method, introducing an additional eccentricity to the evolution. The

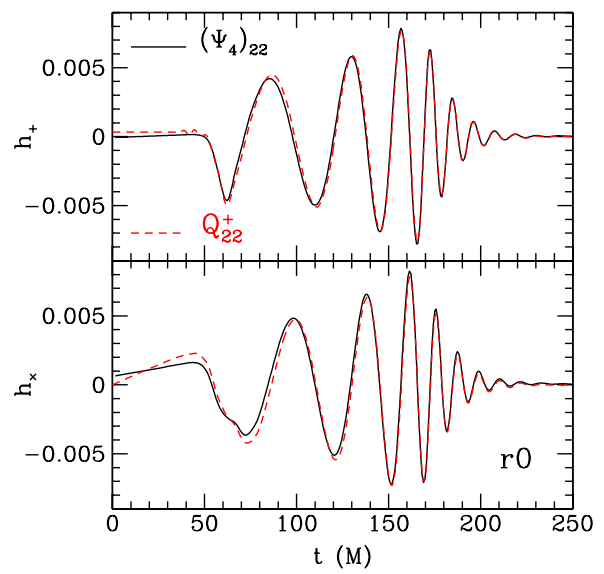

FIG. 14 (color online). Comparison of the two polarization amplitudes $h_{+}$(upper graph) and $h_{\times}$(lower graph) as computed with $\Psi_{4}$ (continuous black line) or with the gauge-invariant quantities $Q_{\ell m}^{+}$(dashed red line). Note the two polarizations are computed using the lowest (and dominant) multipole $\ell=$ $2, m=2$ and are extracted at $r_{\mathrm{E}}=50 \mathrm{M}$. 

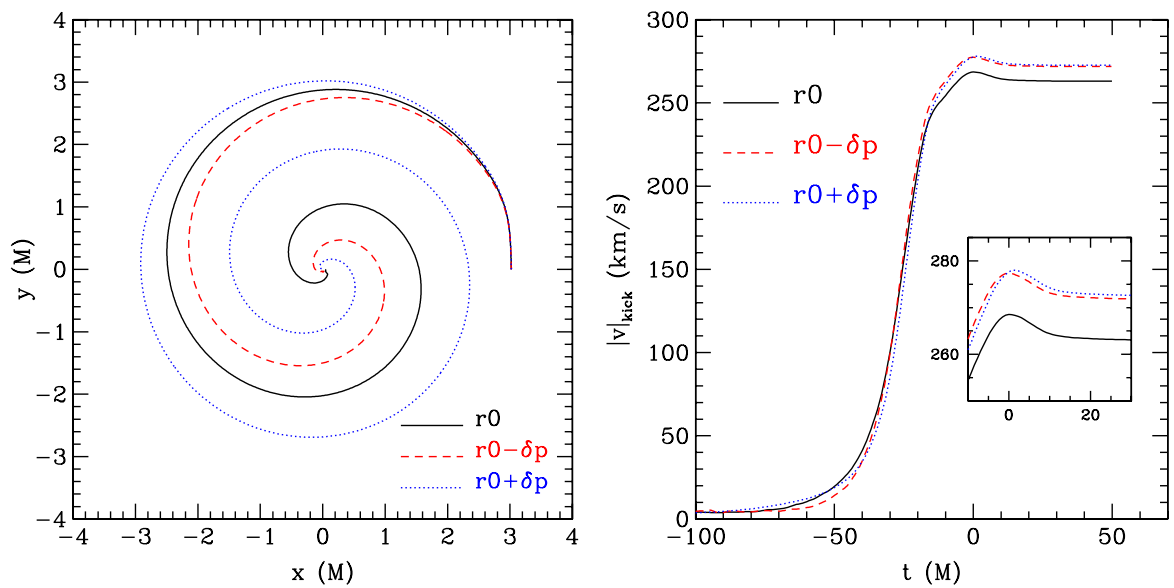

FIG. 15 (color online). Left panel: Coordinate trajectories for one of the black holes for the $r 0$ compared with similar models where the initial linear momenta have been changed by $\pm 3 \%$ in order to modify the eccentricity of the inspiral. Right panel: Recoil velocity for the $r 0$ case is compared with similar models for which the initial eccentricity has been increased by adding and subtracting $3 \%$ of the initial linear momentum of the black holes relative to the $r 0$ values. The effect of increased eccentricity in the final merger is to increase the size of the kick, by about $4 \%$ in both cases.

resulting black hole trajectories and kick determinations are shown, respectively, in Fig. 15. We see that although the level of applied eccentricity is large, and in fact much larger than the expected eccentricity due to the intrinsic inaccuracy of the effective potential method, it modifies the recoil by only about $10 \mathrm{~km} / \mathrm{s}$, that is, $4 \%$. Further, in both the high and low energy cases, the recoil is increased over the fiducial $r 0$ case, suggesting that increased eccentricity generically leads to a slightly larger recoil.
[1] A. Peres, Phys. Rev. 128, 2471 (1962).

[2] J. D. Bekenstein, Phys. Rev. D 7, 949 (1973).

[3] M.J. Fitchett, Mon. Not. R. Astron. Soc. 203, 1049 (1983).

[4] M. J. Fitchett and S. Detweiler, Mon. Not. R. Astron. Soc. 211, 933 (1984).

[5] M. Favata, S. A. Hughes, and D. E. Holz, Astrophys. J. 607, L5 (2004).

[6] F. Herrmann, D. Shoemaker, and P. Laguna, arXiv:gr-qc/ 0601026.

[7] J. G. Baker et al., Astrophys. J. 653, L93 (2006).

[8] J. A. Gonzalez, U. Sperhake, B. Bruegmann, M. Hannam, and S. Husa, Phys. Rev. Lett. 98, 091101 (2007).

[9] F. Herrmann, I. Hinder, D. Shoemaker, P. Laguna, and R. A. Matzner, arXiv:gr-qc/0701143.

[10] M. Koppitz, D. Pollney, C. Reisswig, L. Rezzolla, J. Thornburg, P. Diener, and E. Schnetter, Phys. Rev. Lett. 99, 041102 (2007).

[11] J.A. Gonzalez, M.D. Hannam, U. Sperhake, B. Brugmann, and S. Husa, Phys. Rev. Lett. 98, 231101 (2007).

[12] M. Campanelli, C. O. Lousto, Y. Zlochower, and D. Merritt, Astrophys. J. 659, L5 (2007).

[13] M. Campanelli, C. O. Lousto, Y. Zlochower, and D. Merritt, Phys. Rev. Lett. 98, 231102 (2007).

[14] B. Bruegmann, J. Gonzalez, M. Hannam, S. Husa, and U. Sperhake, arXiv:0707.0135.
[15] J. D. Schnittman, Phys. Rev. D 70, 124020 (2004).

[16] T. Bogdanovic, C.S. Reynolds, and M.C. Miller, arXiv:astro-ph/0703054.

[17] T. Goodale, G. Allen, G. Lanfermann, J. Massó, T. Radke, E. Seidel, and J. Shalf, in 5th International Conference on Vector and Parallel Processing - VECPAR'2002, Lecture Notes in Computer Science (Springer, Berlin, 2003).

[18] Cactus Computational Toolkit, http://www. cactuscode.org.

[19] M. Alcubierre, B. Brügmann, T. Dramlitsch, J. A. Font, P. Papadopoulos, E. Seidel, N. Stergioulas, and R. Takahashi, Phys. Rev. D 62, 044034 (2000).

[20] M. Alcubierre, B. Brügmann, P. Diener, M. Koppitz, D. Pollney, E. Seidel, and R. Takahashi, Phys. Rev. D 67, 084023 (2003).

[21] M. Alcubierre, B. Brügmann, P. Diener, F. S. Guzmán, I. Hawke, S. Hawley, F. Herrmann, M. Koppitz, D. Pollney, and E. Seidel et al., Phys. Rev. D 72, 044004 (2005); http://link.aps.org/abstract/PRD/v72/e044004.

[22] J. G. Baker, J. Centrella, D.-I. Choi, M. Koppitz, and J. van Meter, Phys. Rev. D 73, 104002 (2006).

[23] M. Campanelli, C. O. Lousto, P. Marronetti, and Y. Zlochower, Phys. Rev. Lett. 96, 111101 (2006).

[24] T. Nakamura, K. Oohara, and Y. Kojima, Prog. Theor. Phys. Suppl. 90, 1 (1987).

[25] M. Shibata and T. Nakamura, Phys. Rev. D 52, 5428 (1995). 
[26] T.W. Baumgarte and S.L. Shapiro, Phys. Rev. D 59, 024007 (1998).

[27] J. W. York, in Sources of Gravitational Radiation, edited by L. L. Smarr (Cambridge University Press, Cambridge, England1979), ISBN 0-521-22778-X, pp. 83-126.

[28] C. W. Misner, K. S. Thorne, and J. A. Wheeler, Gravitation (W. H. Freeman, San Francisco, 1973).

[29] J. G. Baker, J. Centrella, D.-I. Choi, M. Koppitz, and J. van Meter, Phys. Rev. Lett. 96, 111102 (2006).

[30] J. van Meter, J. G. Baker, M. Koppitz, and D.-I. Choi, Phys. Rev. D 73, 124011 (2006).

[31] E. Schnetter, S.H. Hawley, and I. Hawke, Classical Quantum Gravity 21, 1465 (2004).

[32] Mesh Refinement with Carpet, http://www.carpetcode. org/.

[33] J. Thornburg, Phys. Rev. D 54, 4899 (1996).

[34] J. Thornburg, Classical Quantum Gravity 21, 743 (2004).

[35] S. Brandt and B. Brügmann, Phys. Rev. Lett. 78, 3606 (1997).

[36] M. Ansorg, B. Brügmann, and W. Tichy, Phys. Rev. D 70, 064011 (2004).

[37] L. L. Smarr, Phys. Rev. Lett. 30, 71 (1973).

[38] D. Christodoulou, Phys. Rev. Lett. 25, 1596 (1970).

[39] G. B. Cook, Phys. Rev. D 50, 5025 (1994).

[40] H. P. Pfeiffer, S. A. Teukolsky, and G. B. Cook, Phys. Rev. D 62, 104018 (2000).

[41] G. B. Cook, Living Rev. Relativity 3, 5 (2000), http:// www.livingreviews.org/lrr-2000-5.

[42] J. Thornburg, Classical Quantum Gravity 21, 743 (2004), http://stacks.iop.org/0264-9381/21/743.

[43] L. Gunnarsen, H. Shinkai, and K. Maeda, Classical Quantum Gravity 12, 133 (1995).

[44] L. Lehner and O. M. Moreschi, arXiv:0706.1319.

[45] S. A. Teukolsky, Astrophys. J. 185, 635 (1973).

[46] M. Campanelli and C. O. Lousto, Phys. Rev. D 59, 124022 (1999).

[47] J. G. Baker, J. Centrella, D.-I. Choi, M. Koppitz, J. van Meter, and M. C. Miller, Astrophys. J. 653, L93 (2006).

[48] J. A. González, U. Sperhake, B. Brügmann, M. Hannam, and S. Husa, Phys. Rev. Lett. 98, 091101 (2007).

[49] M. Koppitz et al., arXiv:gr-qc/0701163.

[50] G. Allen, K. Camarda, and E. Seidel, arXiv:gr-qc/ 9806036.

[51] M.E. Rupright, A. M. Abrahams, and L. Rezzolla, Phys. Rev. D 58, 044005 (1998).

[52] K. Camarda and E. Seidel, Phys. Rev. D 59, 064019
(1999).

[53] V. Moncrief, Ann. Phys. (N.Y.) 88, 323 (1974).

[54] A. Abrahams and R. Price, Phys. Rev. D 53, 163 (1996).

[55] A. Nagar and L. Rezzolla, Classical Quantum Gravity 22, R167 (2005); 23, 4297(E) (2006).

[56] L. Rezzolla, A. M. Abrahams, R. A. Matzner, M.E. Rupright, and S.L. Shapiro, Phys. Rev. D 59, 064001 (1999).

[57] J. Baker, S. R. Brandt, M. Campanelli, C. O. Lousto, E. Seidel, and R. Takahashi, Phys. Rev. D 62, 127701 (2000).

[58] J. A. Font, T. Goodale, S. Iyer, M. Miller, L. Rezzolla, E. Seidel, N. Stergioulas, W.-M. Suen, and M. Tobias, Phys. Rev. D 65, 084024 (2002).

[59] K. Thorne, Rev. Mod. Phys. 52, 299 (1980).

[60] N. Y. C. F. Sopuerta and P. Laguna, Phys. Rev. D 74, 124010 (2006); 75, 069903(E) (2007).

[61] T. Damour and A. Gopakumar, Phys. Rev. D 73, 124006 (2006).

[62] The presence of a small eccentricity prevents from a strict monotonicity of the recoil velocity for binaries starting from a large separation. In this case, very small oscillations appear over the orbital timescale.

[63] L. E. Kidder, Phys. Rev. D 52, 821 (1995).

[64] O. Dreyer, B. Krishnan, D. Shoemaker, and E. Schnetter, Phys. Rev. D 67, 024018 (2003); http://link.aps.org/ abstract/PRD/v67/e024018.

[65] A. Ashtekar and B. Krishnan, Phys. Rev. D 68, 104030 (2003).

[66] E. Schnetter, B. Krishnan, and F. Beyer, Phys. Rev. D 74, 024028 (2006).

[67] M. Alcubierre, B. Brügmann, P. Diener, F. S. Guzmán, I. Hawke, S. Hawley, F. Herrmann, M. Koppitz, D. Pollney, E. Seidel et al., Phys. Rev. D 72, 044004 (2005).

[68] E. Seidel, Prog. Theor. Phys. Suppl. 136, 87 (1999); http:// ptp.ipap.jp/link?PTPS/136/87/.

[69] S. Brandt and E. Seidel, Phys. Rev. D 52, 870 (1995); http://link.aps.org/abstract/PRD/v52/p870.

[70] E. Poisson, Phys. Rev. D 70, 084044 (2004).

[71] K. Martel and E. Poisson, Phys. Rev. D 71, 104003 (2005).

[72] G. Kupi, P. Amaro-Seoane, and R. Spurzem, Mon. Not. R. Astron. Soc. 371, L45 (2006).

[73] C. T. Cunningham, R.H. Price, and V. Moncrief, Astrophys. J. 224, 643 (1978).

[74] C. Cunningham, R. Price, and V. Moncrief, Astrophys. J. 230, 870 (1979). 